\title{
Inverse Problem Reveals Conditions for Characteristic Retinal Degeneration Patterns in Retinitis Pigmentosa under the Trophic Factor Hypothesis
}

\author{
Paul A. Roberts ${ }^{1, *}$ \\ ${ }^{1}$ Baden Lab, Centre for Sensory Neuroscience and Computation, School of Life \\ Sciences, University of Sussex, Brighton, UK
}

Correspondence*:

Paul A. Roberts

p.a.roberts@univ.oxon.org

\section{ABSTRACT}

Retinitis pigmentosa $(\mathrm{RP})$ is the most common inherited retinal dystrophy with a prevalence of about 1 in 4000, affecting approximately 1.5 million people worldwide. Patients with RP experience progressive visual field loss as the retina degenerates, destroying light-sensitive photoreceptor cells (rods and cones), with rods affected earlier and more severely than cones. Spatio-temporal patterns of retinal degeneration in human RP have been well characterised; however, the mechanism(s) giving rise to these patterns have not been conclusively determined. One such mechanism, which has received a wealth of experimental support, is described by the trophic factor hypothesis. This hypothesis suggests that rods produce a trophic factor necessary for cone survival; the loss of rods depletes this factor, leading to cone degeneration. In this paper we formulate a partial differential equation mathematical model of RP in one spatial dimension, spanning the region between the retinal centre (fovea) and the retinal edge (ora serrata). Using this model we derive and solve an inverse problem, revealing for the first time experimentally testable conditions under which the trophic factor mechanism will qualitatively recapitulate the spatio-temporal patterns of retinal regeneration observed in human RP.

Keywords: Partial Differential Equations, Asymptotic Analysis, Retina, Photoreceptors, Rod-derived Cone Viability Factor

\section{INTRODUCTION}

The group of inherited retinal diseases known as retinitis pigmentosa (RP) causes the progressive loss of visual function (Hamel, 2006; Hartong et al., 2006). The patterns of visual field loss associated with the human version of this condition have been well characterised (Grover et al., 1998); however, the mechanisms underpinning these patterns have yet to be conclusively determined (Newton and Megaw, 2020). In this paper, we use mathematical models to predict the conditions under which a trophic factor mechanism could explain these patterns.

The retina is a tissue layer lining the back of the eye containing light-sensitive cells known as photoreceptors, which come in two varieties: rods and cones (Fig. 1A). Rods confer monochromatic vision under low-light (scotopic) conditions, while cones confer colour vision under well-lit (photopic) conditions (Oyster, 1999). In RP, rod function and health are typically affected earlier and more severely than those of cones, with cone loss following rod loss. Rods are lost since either they or the neighbouring retinal 
bioRxiv preprint doi: https://doi.org/10.1101/2021.09.04.458968; this version posted February 14, 2022. The copyright holder for this preprint (which was not certified by peer review) is the author/funder, who has granted bioRxiv a license to display the preprint in perpetuity. It is made available under aCC-BY-NC-ND 4.0 International license.

pigment epithelium express a mutant version of one or both alleles (depending on inheritance mode) of a gene associated with RP. It is hypothesised that cones are lost following rods since they depend upon rods either directly or indirectly for their survival (Daiger et al., 2007; Hamel, 2006; Hartong et al., 2006).

A number of mechanisms have been hypothesised to explain secondary cone loss, including trophic factor (TF) depletion (Aït-Ali et al., 2015; Léveillard et al., 2004; Mei et al., 2016), oxygen toxicity (Stone et al., 1999; Travis et al., 1991; Valter et al., 1998), metabolic dysregulation (Punzo et al., 2009, 2012), toxic substances (Ripps, 2002) and microglia (Gupta et al., 2003). While not typically related to spatio-temporal patterns of retinal degeneration in the literature, it is reasonable to infer that these mechanisms play an important role in determining spatio-temporal patterns of retinal degeneration.

Grover et al. (1998) have classified the spatio-temporal patterns of visual field loss in RP patients into three patterns and six sub-patterns (see Fig. 2). Pattern 1A consists in a restriction of the peripheral visual field, while Pattern 1B also includes a para-/peri-foveal ring scotoma (blind spot); Pattern 2 (A, B and C) involves an initial loss of the superior visual field, winding nasally or temporally into the inferior visual field; lastly, Pattern 3 starts with loss of the mid-peripheral visual field, before spreading into the superior or inferior visual field and winding around the far-periphery. In all cases central vision is the best preserved, though it too is eventually lost (Hamel, 2006; Hartong et al., 2006). Patterns of visual field loss and photoreceptor degeneration (cell loss) are directly related (Escher et al., 2012), loss of the superior visual field corresponding to degeneration of photoreceptors in the inferior retina and vice versa, and loss of the temporal visual field corresponding to degeneration of photoreceptors in the nasal retina and vice versa.

In this paper we explore the conditions under which the TF mechanism, in isolation, can replicate the patterns of cone degeneration observed in vivo. Isolating a mechanism in this way enables us to identify the effects for which it is sufficient to account, avoiding confusion with other mechanistic causes. Understanding the mechanisms of secondary cone degeneration is important since it is the cones that provide high-acuity colour vision, and hence their loss, rather than the preceding rod loss, which is the most debilitating. Therefore, by elucidating these mechanisms, we can develop targeted therapies to prevent or delay cone loss, preserving visual function. The TF mechanism has been studied in detail. Rod photoreceptors have been shown to produce a TF called rod-derived cone viability factor (RdCVF), which is necessary for cone survival (Fintz et al., 2003; Léveillard et al., 2004; Mohand-Saïd et al., 1998, 2000, 1997; Yang et al. 2009). RdCVF increases cone glucose uptake, and hence aerobic glycolysis, by binding to the cone transmembrane protein Basigin-1, which consequently binds to the glucose transporter GLUT1 (Ait-Ali et al., 2015). Cones do not produce RdCVF, thus, when rods are lost, RdCVF concentration drops and cone degeneration follows (though it has been suggested that it may ultimately be oxygen toxicity which kills cones; Léveillard and Sahel, 2017).

Thus far, two groups have developed mathematical models operating under the TF hypothesis. Camacho et al. have developed a series of (non-spatial) dynamical systems ordinary differential equation models to describe the role of RdCVF in health and RP (Colón Vélez et al., 2003; Camacho et al., 2010; Camacho and Wirkus, 2013, Camacho et al., 2014, 2016a b c, 2019, 2020, 2021; Wifvat et al., 2021). In Roberts (2022), we developed the first partial differential equation (PDE) models of the TF mechanism in RP, predicting the spatial spread of retinal degeneration. It was found that, assuming all cones are equally susceptible to RdCVF deprivation and that rods degenerate exponentially with a fixed decay rate, the mechanism is unable to replicate in vivo patterns of retinal degeneration. Previous modelling studies have also considered the oxygen toxicity (Roberts et al., 2017, 2018 and related Roberts et al., 2016b) and toxic substance (Burns et al., 2002) mechanisms, predicting the spatio-temporal patterns of retinal degeneration they would 
generate. For a review of these and other mathematical models of the retina in health, development and disease see Roberts et al. (2016a).

In this study, we extend our work in Roberts (2022) by formulating and solving an inverse problem to determine the spatially heterogeneous cone susceptibility to RdCVF deprivation and rod exponential decay rate profiles that are required to qualitatively recapitulate observed patterns of spatio-temporal degeneration in human RP.

\section{MATERIAL AND METHODS}

\subsection{Model Formulation}

We begin by formulating a reaction-diffusion PDE mathematical model (a simplified version of the model presented in Roberts, 2022), posing it on a spherical geometry to replicate that of the human retina. This geometry is most naturally represented using a spherical polar coordinate system, $(r, \theta, \phi)$, centred in the middle of the vitreous body, where $r \geq 0(\mathrm{~m})$ is the distance from the origin, $0 \leq \theta \leq \pi$ (rad) is the polar angle and $0 \leq \phi<2 \pi(\mathrm{rad})$ is the azimuthal angle. To create a more mathematically tractable model, we simplify the geometry by assuming symmetry about the $z$-axis (directed outward from the origin through the foveal centre), eliminating variation in the azimuthal direction, and effectively depth-average through the retina, assuming that it lies at a single fixed distance, $R>0(\mathrm{~m})$, from the origin at all eccentricities, $\theta$, leveraging the fact that the retinal width is two orders of magnitude smaller than the eye's radius (Oyster, 1999). Thus, we have reduced the coordinate system to $(R, \theta)$, where $R$ is a positive constant parameter and $0 \leq \theta \leq \Theta$ is an independent variable, which we bound to range between the fovea (at $\theta=0 \mathrm{rad}$ ) and the ora serrata (at $\theta=\Theta=1.33 \mathrm{rad}$; see Fig. 11A). We further simplify the model by non-dimensionalising; scaling the dependent and independent variables so that they and the resultant model parameters are dimensionless and hence unitless. This reduces the number of parameters (including eliminating $R$ ) and allows us to identify the dominant terms of the governing equations in the ensuing asymptotic analysis. For this reason, there are no units to be stated in Figs. $3-10$. For the full dimensional model and non-dimensionalisation see Roberts (2022).

We proceed directly to the dimensionless model, which consists of a system of PDEs in terms of the dependent variables: TF concentration, $f(\theta, t)$, rod photoreceptor density, $p_{r}(\theta, t)$, and cone photoreceptor density, $p_{c}(\theta, t)$; as functions of the independent variables: polar angle, scaled to lie in the range $0 \leq \theta \leq 1$, and time, $t>0$ (see Table 1 ).

The TF equation is as follows

$$
\frac{\partial f}{\partial t}=\underbrace{\frac{D_{f}}{\sin (\Theta \theta)} \frac{\partial}{\partial \theta}\left(\sin (\Theta \theta) \frac{\partial f}{\partial \theta}\right)}_{\text {diffusion }}+\underbrace{\alpha p_{r}}_{\text {production }}-\underbrace{\beta f p_{c}}_{\text {consumption }}-\underbrace{\eta f}_{\text {decay }},
$$

where the parameters, $D_{f}$, the TF diffusivity, $\alpha$, the rate of $\mathrm{TF}$ production by rods, $\beta$, the rate of $\mathrm{TF}$ consumption by cones, and $\eta$, the rate of TF decay, are positive constants. Trophic factor is free to diffuse across the retina through the interphotoreceptor matrix (Aït-Ali et al., 2015), and is assumed to be produced by rods at a rate proportional to their local density, to be consumed by cones at a rate proportional the product of the local TF concentration and the local cone density, and to decay at a rate proportional to the 
107 local TF concentration. The rod equation takes the following form

$$
\frac{\partial p_{r}}{\partial t}=-\underbrace{\phi_{r}(\theta) p_{r}}_{\begin{array}{c}
\text { cell degeneration } \\
\text { (mutation-induced) }
\end{array}},
$$

108 where we allow the variable $\phi_{r}(\theta)$, the rate of mutation-induced rod degeneration, to vary spatially 109 (functional forms defined in the Results section), or take a constant value, $\phi_{r}$. Rods degenerate due to their 110 expression of a mutant gene (Hamel, 2006, Hartong et al., 2006) at a rate assumed to be proportional to 111 their local density and independent of the TF concentration (Aï-Ali et al., 2015). We note that Eqn. (2) 112 can be solved to yield $p_{r}(\theta, t)=p_{r_{\text {init }}}(\theta) e^{-\phi_{r}(\theta) t}$ (where $p_{r_{\text {init }}}(\theta)$, the initial value of $p_{r}(\theta, t)$, is defined below), provided there is no delay in onset or interruption of degeneration. The cone equation is as follows

$$
\frac{\partial p_{c}}{\partial t}=-\underbrace{p_{c} \lambda_{2}(f)}_{\begin{array}{c}
\text { cell degeneration } \\
\text { (TF starvation) }
\end{array}} .
$$

Defining the Heaviside step function, $H(\cdot)$, such that

$$
H(x):= \begin{cases}0 & \text { if } x<0 \\ 1 & \text { if } x \geq 0\end{cases}
$$

the function $\lambda_{2}(f)$ is given by

$$
\lambda_{2}(f)=1-H\left(f-f_{\text {crit }}(\theta)\right),
$$

114 where we allow the variable $f_{\text {crit }}(\theta)$, the TF threshold concentration, to vary spatially (functional forms 115 defined in the Results section), or take a constant value, $f_{\text {crit }}$. Cone density is assumed to remain constant 116 provided the local TF concentration, $f(\theta, t)$, remains in the healthy range at or above the critical threshold, $117 f_{\text {crit }}$, while cones are assumed to decay (due to TF starvation) at a rate proportional to their local density if $118 f(\theta, t)$ drops below this threshold.

Having defined the governing equations (Eqs. (1)-(3)), we close the system by imposing boundary and initial conditions. We apply zero-flux boundary conditions at both ends of the domain,

$$
\frac{\partial f}{\partial \theta}(0, t)=0=\frac{\partial f}{\partial \theta}(1, t)
$$

such that there is no net flow of TF into or out of the domain. This is justified by symmetry at $\theta=0$, while we assume that TF cannot escape from the retina where it terminates at the ora serrata $(\theta=1)$. The healthy rod and cone distributions are given by the following functions

$$
\begin{aligned}
& \tilde{p}_{r}(\theta)=B_{3} \theta e^{-b_{3} \theta}, \\
& \tilde{p}_{c}(\theta)=B_{1} e^{-b_{1} \theta}+B_{2} e^{-b_{2} \theta},
\end{aligned}
$$

121 where the values of the positive constants $B_{1}, B_{2}, B_{3}, b_{1}, b_{2}$ and $b_{3}$ are found by fitting to the mean of Curcio et al. (1990)'s measurements of healthy human rod and cone distributions along the temporal horizontal meridian using the Trust-Region Reflective algorithm in Matlab's curve fitting toolbox (see Fig. 
124

13). Lastly, we impose the initial conditions

$$
f(\theta, 0)=f_{\text {init }}(\theta), \quad p_{r}(\theta, 0)=p_{r_{\text {init }}}(\theta)=\tilde{p}_{r}(\theta), \quad p_{c}(\theta, 0)=p_{c_{\text {init }}}(\theta)=\tilde{p}_{c}(\theta),
$$

where $f_{\text {init }}(\theta)$ is the steady-state solution to Eqs. (1) and (4) with $p_{r}=p_{r_{\text {init }}}(\theta)$ and $p_{c}=p_{c_{\text {init }}}(\theta)$ (see Fig. 3A). Thus, the retina starts in the healthy state in all simulations. See Table 2 for the dimensionless parameter values (see Roberts, 2022, for dimensional values and justification of parameter values). The model presented here simplifies that in Roberts (2022) in the following ways: it does not include treatment, cone outer segment regeneration, or initial patches of rod or cone loss, while mutation-induced rod loss is active for all simulations in this study. The present model also adds two new features to the previous model: allowing the rate of mutation-induced rod degeneration, $\phi_{r}(\theta)$, and the TF threshold concentration, $f_{\text {crit }}(\theta)$, to vary spatially, where before they were constant (or piecewise constant in the high $f_{\text {crit }}$ subcase).

\subsection{Numerical Solutions}

Numerical (computational) solutions to Eqs. (1)-(5) were obtained using the method of lines (as in Roberts, 2022), discretising in space and then integrating in time. The time integration was performed using the Matlab routine ode15s, a variable-step, variable-order solver, designed to solve problems involving multiple timescales such as this (Matlab version R2020a was used here and throughout the paper). We used a relative error tolerance of $10^{-6}$ and an absolute error tolerance of $10^{-10}$, with the remaining settings at their default values. The number of spatial mesh points employed varies between simulations, taking values of 26, 51, 101, 401 or 4001 . The upper bound of 4001 mesh points was chosen such that the distance between mesh points corresponds to the average width of a photoreceptor. In each case the maximum computationally feasible mesh density was employed, all mesh densities being sufficient to achieve accurate results. The initial TF profile, $f(\theta, 0)=f_{\text {init }}(\theta)$, was calculated by discretising Eqs. (1) and (4) at steady-state, using a finite difference scheme, and solving the consequent system of nonlinear algebraic equations using the Matlab routine fsolve (which employs a Trust-Region-Dogleg algorithm) with $p_{r}=p_{r_{\text {init }}}(\theta)$ and $p_{c}=p_{c_{\text {init }}}(\theta)$.

\subsection{Inverse Problem}

Our previous modelling study of the TF hypothesis predicted patterns of cone degeneration which failed to match any known patterns in human RP (Roberts, 2022). In that study we made the simplifying assumption that model parameters are spatially uniform, such that they do not vary with retinal eccentricity. While this is a reasonable assumption in most cases, we have reason to believe that two of the parameters - the rate of mutation-induced rod loss, $\phi_{r}$, and the TF threshold concentration, $f_{\text {crit }}$ - may vary spatially, which could help account for in vivo patterns of retinal degeneration. Curcio et al.(1993) measured the rate of decay of rod photoreceptors at a range of eccentricities in healthy human retinas. They found that this rate varies with eccentricity, degeneration occurring most rapidly in the central retina. While similar measurements have not, to the best of our knowledge, been made in the RP retina, it is reasonable to assume that the rate of rod loss would also be heterogeneous in this case, quite possibly with a different heterogeneity than in the healthy case. Further, under healthy conditions, the RdCVF concentration at the centre of the retina (near $\theta=0)$ is much lower $\left(f(\theta, t) \sim O\left(10^{-5}\right)\right)$ than in the remainder of the retina (where $f(\theta, t) \sim O(0.1)$ to $O(1)$, see Fig. 3A). Therefore, it is reasonable to assume that central retinal cones are able to cope with lower RdCVF concentrations than those toward the periphery, and hence that $f_{\text {crit }}$ is also heterogeneous. To determine whether these heterogeneities could account for cone degeneration patterns in human RP, we formulate and solve something known as an inverse problem. 
bioRxiv preprint doi: https://doi.org/10.1101/2021.09.04.458968; this version posted February 14, 2022. The copyright holder for this preprint (which was not certified by peer review) is the author/funder, who has granted bioRxiv a license to display the preprint in perpetuity. It is made available under aCC-BY-NC-ND 4.0 International license.

In an inverse problem we seek to determine the model input required to attain a known/desired output. In this case the known output is the target cone degeneration profile, $t_{\text {degen }}(\theta)$, while the input is either the rate of mutation-induced rod loss profile, $\phi_{r}(\theta)$, or the TF threshold concentration profile, $f_{\text {crit }}(\theta)$, with corresponding inverses denoted as $\phi_{r}(\theta)=\phi_{r_{\text {inv }}}(\theta)$ and $f_{\text {crit }}(\theta)=f_{\text {crit }_{\text {inv }}}(\theta)$ respectively. When searching for $\phi_{r_{\text {inv }}}(\theta)$, we hold the TF threshold concentration constant at $f_{\text {crit }}(\theta)=f_{\text {crit }}=3 \times 10^{-5}$, while, when searching for $f_{\text {critinv }}(\theta)$, we hold the rate of mutation-induced rod loss constant at $\phi_{r}(\theta)=$ $\phi_{r}=7.33 \times 10^{-2}$. The constant value of $f_{\text {crit }}$ is chosen to lie just below the minimum steady-state value of $f(\theta)$, such that, in the absence of rod loss, cones remain healthy, while the constant value of $\phi_{r}$ is chosen to be one hundred times higher than the value that can be inferred from measurements in the healthy human retina (Curcio et al., 1993), placing the timescale of the resultant cone loss on the order of decades, in agreement with in vivo RP progression rates (Hamel, 2006; Hartong et al., 2006).

We consider a range of target cone degeneration profiles, summarised in Table 3 and Fig. 5, which qualitatively replicate visual field loss Patterns 1A, 1B and 3 seen in vivo (and hence the corresponding in vivo cone degeneration patterns; taking the degeneration of the far-peripheral retina to occur in a radially symmetric manner in Pattern 3 - see Fig. 2 and Grover et al., 1998). We do not consider patterns of type 2 (to be explored in a future study) as these cannot be replicated by a 1D model (since the radial symmetry, assumed by the 1D model, is broken by variation in the azimuthal/circumferential direction). For each degeneration pattern we consider a set of sub-patterns to examine how this affects the shape of the inverses, allowing us to confirm that a modest change in the degeneration pattern results in a modest change in the inverses, while exploring both linear/piecewise linear profiles and more biologically realistic nonlinear (quadratic/cubic/exponential) patterns. We also consider a uniform target cone degeneration profile for comparison.

For each pattern, we consider the effect of two (biologically realistic) scalings for the rate of TF production by rods, $\alpha$, and the rate of TF consumption by cones, $\beta$, upon the inverse profiles: (i) Scaling 1 - for which $\alpha=7.01 \times 10^{4}$ and $\beta=1.79 \times 10^{6}$ as in Roberts (2022); and (ii) Scaling 2 - for which $\alpha=7.01 \times 10^{2}$ and $\beta=1.79 \times 10^{4}$. Under Scaling 1 , production and consumption of TF dominate over decay (with rate constant $\eta$ ), such that decay has a negligible effect upon the TF profile and model behaviour. Under Scaling 2, TF production and consumption occur at a similar rate to decay, such that they balance each other, resulting in a different TF profile and model behaviour (see Fig. 3A and C). As discussed in Roberts (2022), none of $\alpha, \beta$ or $\eta$ have been measured. The decay rate, $\eta$, was chosen to match the measured decay rate of proteins in living human cells (Eden et al. 2011). Under Scaling 1, the consumption rate, $\beta$, is chosen such that it dominates over the decay rate (being a factor $\epsilon^{-1}=O\left(10^{2}\right)$ larger), while the production rate, $\alpha$, is chosen to balance consumption (see the Analytical Inverse section). This is a sensible scaling as it is likely that cones consume RdCVF at a much faster rate than that at which it decays. It is possible, however, that cones consume RdCVF at a similar rate to its decay rate, which is the scenario we consider in Scaling 2; reducing $\alpha$ and $\beta$ by a factor of $100\left(\sim \epsilon^{-1}\right)$ to bring consumption and production into balance with decay (see the Analytical Inverse section).

We solve the inverse problem both analytically and numerically (computationally), as described in the Analytical Inverse and Numerical Inverse sections below. Analytical approximations are computationally inexpensive and provide deeper insight into model behaviour, while numerical solutions, though computationally intensive, are more accurate. 


\subsubsection{Analytical Inverse}

To derive analytical (algebraic) approximations for the inverses, $\phi_{r_{\text {inv }}}(\theta)$ and $f_{\text {critinv }}(\theta)$, we perform an asymptotic analysis, seeking the leading order behaviour of Eqs. (1)-(5). Proceeding as in Roberts (2022) (where we considered a steady-state problem), we choose $\epsilon=O\left(10^{-2}\right)$ and scale the parameters $\eta=\epsilon^{-1} \eta^{\prime}$ and $b_{1}=\epsilon^{-1} b_{1}^{\prime}$, introducing the new scaling $\phi_{r}(\theta)=\epsilon \phi_{r}^{\prime}(\theta)$, as we study the time-dependent problem here (where dashes ' denote scaled variables and parameters). We consider two possible (biologically realistic) scalings on $\alpha$ and $\beta$ : (i) Scaling 1 - for which $\alpha=\epsilon^{-2} \alpha^{\prime}$ and $\beta=\epsilon^{-3} \beta^{\prime}$ as in Roberts (2022) 215 (corresponding to $\alpha=7.01 \times 10^{4}$ and $\beta=1.79 \times 10^{6}$ ); and (ii) Scaling $2-$ for which $\alpha=\epsilon^{-1} \alpha^{\prime}$ and $216 \beta=\epsilon^{-2} \beta^{\prime}$ (corresponding to $\alpha=7.01 \times 10^{2}$ and $\beta=1.79 \times 10^{4}$ ). All remaining parameters are assumed 217 to be $O(1)$. We also scale the dependent variable $p_{c}(\theta, t)=\epsilon p_{c}^{\prime}(\theta, t)$, and assume $f(\theta, t)=O(1)$ and $218 p_{r}(\theta, t)=O(1)$.

Applying the above scalings and dropping the dashes (working with the scaled versions of the variables and parameters, but omitting the dashes ' for notational convenience), Eqn. (2) becomes

$$
\frac{\partial p_{r}}{\partial t}=-\epsilon \phi_{r}(\theta) p_{r}
$$

Thus, on this (fast) timescale, the rod density is constant. Since we are interested in the timescale upon which rods degenerate, we scale time as $t^{\prime}=\epsilon t$ such that the decay term enters the dominant balance. Thus, on this slow timescale, after dropping the dashes, we have that

$$
\frac{\partial p_{r}}{\partial t}=-\phi_{r}(\theta) p_{r}
$$

219 such that, at leading order, $p_{r_{0}}(\theta, t)=p_{r_{\text {init }_{0}}}(\theta) e^{-\phi_{r}(\theta) t}=B_{3} \theta e^{-b_{3} \theta} e^{\phi_{r}(\theta) t}$.

We are interested here in the regime in which cones have not yet degenerated, thus we assume the leading order cone density remains constant at $p_{c_{0}}(\theta)=p_{c_{\text {init }_{0}}}(\theta)=B_{2} e^{-b_{2} \theta}$.

Applying Scaling 1 and the slow timescale to Eqn. (1) we obtain

$$
\epsilon \frac{\partial f}{\partial t}=D_{f} \frac{\partial^{2} f}{\partial \theta^{2}}+D_{f} \Theta \cot (\Theta \theta) \frac{\partial f}{\partial \theta}+\epsilon^{-2} \alpha p_{r}-\epsilon^{-2} \beta p_{c} f-\epsilon^{-1} \eta f .
$$

Since the TF dynamics occur on a faster timescale than mutation-induced rod loss, we make a quasi-steadystate approximation (QSSA), assuming that the TF concentration instantaneously takes its steady-state profile, for any given rod density profile, as the rods degenerate $\left(\epsilon \partial_{t} f \sim 0\right)$. Thus, at leading order, we obtain

$$
f_{0_{\mathrm{QSSA}}}(\theta)=\frac{\alpha p_{r_{0}}(\theta, t)}{\beta p_{c_{0}}(\theta)}
$$

222 Rearranging this expression and assuming that cone degeneration initiates when $f_{0_{\mathrm{QSSA}}}(\theta)=f_{\text {crit }}(\theta)$, we obtain the cone degeneration time profile,

$$
t_{\text {degen }}(\theta)=\frac{1}{\phi_{r}(\theta)}\left(\log \left(\frac{\alpha B_{3}}{\beta B_{2} f_{\text {crit }}(\theta)} \theta\right)-\left(b_{3}-b_{2}\right) \theta\right),
$$


224 the inverse mutation-induced rod degeneration rate profile,

$$
\phi_{r_{\text {inv }}}(\theta)=\frac{1}{t_{\text {degen }}(\theta)}\left(\log \left(\frac{\alpha B_{3}}{\beta B_{2} f_{\text {crit }}} \theta\right)-\left(b_{3}-b_{2}\right) \theta\right)
$$

and the inverse TF threshold concentration profile,

$$
f_{\text {crit }_{\text {inv }}}(\theta)=\frac{\alpha B_{3}}{\beta B_{2}} \theta e^{-\left(\left(b_{3}-b_{2}\right) \theta+\phi_{r} t_{\text {degen }}(\theta)\right)} .
$$

Alternatively, if we apply Scaling 2 and the slow timescale to Eqn. (1) we obtain

$$
\epsilon \frac{\partial f}{\partial t}=D_{f} \frac{\partial^{2} f}{\partial \theta^{2}}+D_{f} \Theta \cot (\Theta \theta) \frac{\partial f}{\partial \theta}+\epsilon^{-1} \alpha p_{r}-\epsilon^{-1} \beta p_{c} f-\epsilon^{-1} \eta f,
$$

with the TF decay term, $\eta f$, now entering the dominant balance. Applying the QSSA and proceeding as above we find

$$
f_{0_{\mathrm{QSSA}}}(\theta)=\frac{\alpha p_{r_{0}}(\theta, t)}{\beta p_{c_{0}}(\theta)+\eta}
$$

226 with cone degeneration time profile,

$$
t_{\text {degen }}(\theta)=\frac{1}{\phi_{r}(\theta)}\left(\log \left(\frac{\alpha B_{3}}{\left(\beta B_{2}+\eta e^{b_{2} \theta}\right) f_{\text {crit }}(\theta)} \theta\right)-\left(b_{3}-b_{2}\right) \theta\right),
$$

227 inverse mutation-induced rod degeneration rate profile,

$$
\phi_{r_{\text {inv }}}(\theta)=\frac{1}{t_{\text {degen }}(\theta)}\left(\log \left(\frac{\alpha B_{3}}{\left(\beta B_{2}+\eta e^{b_{2} \theta}\right) f_{\text {crit }}} \theta\right)-\left(b_{3}-b_{2}\right) \theta\right),
$$

228 and inverse TF threshold concentration profile,

$$
f_{\text {crit }_{\text {inv }}}(\theta)=\frac{\alpha B_{3}}{\left(\beta B_{2}+\eta e^{b_{2} \theta}\right)} \theta e^{-\left(\left(b_{3}-b_{2}\right) \theta+\phi_{r} t_{\text {degen }}(\theta)\right)} .
$$

229 These equations reveal how the inverses, $\phi_{r_{\text {inv }}}(\theta)$ and $f_{\text {critinv }}(\theta)$, are influenced by our choices for fixed values of $f_{\text {crit }}$ and $\phi_{r}$, respectively. As can be seen from Eqs. (7) and (10), $\phi_{r_{\text {inv }}}(\theta)$ is inversely and monotonically related to $f_{\text {crit }}$, such that as $f_{\text {crit }}$ increases, $\phi_{r_{\text {inv }}}(\theta)$ decreases. Similarly, $f_{\text {crit }}(\theta)$ and $\phi_{r}$ are inversely and monotonically related in Eqs. (8) and (11), such that as $\phi_{r}$ increases, $f_{\text {crit }}$ (inv $(\theta)$ decreases. Lastly, as would be expected intuitively, $t_{\text {degen }}(\theta), \phi_{r_{\text {inv }}}(\theta)$ and $f_{\text {crit }}{ }_{\text {inv }}(\theta)$ all increase monotonically with increasing TF production, $\alpha$, and decrease monotonically with increasing TF consumption, $\beta$, and TF decay $\eta$ (Eqs. (6)-(8) and (9)-(11)).

\subsubsection{Numerical Inverse}

The numerical inverse is calculated by repeatedly solving the forward problem (Eqs. (1)-(5)) for different values of the input $\left(\phi_{r}(\theta)\right.$ or $\left.f_{\text {crit }}(\theta)\right)$, with the aim of converging upon the inverse $\left(\phi_{r_{\text {inv }}}(\theta)\right.$ or $\left.f_{\text {critinv }}(\theta)\right)$. To find $\phi_{r_{\text {inv }}}(\theta)$ we use the Matlab routine fminsearch (which uses a simplex search method), while to

41 obtain $f_{\text {crit }_{\text {inv }}}(\theta)$ the Matlab routine patternsearch (which uses an adaptive mesh technique) was found to 42 be more effective. In both cases the objective function was taken as the sum of squares of the difference 


\section{RESULTS}

between the target cone degeneration profile, $t_{\text {degen }}(\theta)$, and the contour described by $p_{c}(\theta, t) / \tilde{p}_{c}(\theta)=0.99$ (along which cone degeneration is deemed to have initiated). Eqs. (1)-(5) were solved at each iteration as described in the Numerical Solutions section. Numerical inverses were calculated only at those locations (eccentricities) where the analytical inverse failed to generate a $t_{\text {degen }}(\theta)$ profile matching the target profile, the analytical inverse being assumed to hold at all other eccentricities.

We begin by calculating the cone degeneration profiles, $t_{\operatorname{degen}}(\theta)$, in the case where both the rate of mutation induced rod degeneration, $\phi_{r}$, and the TF threshold concentration, $f_{\text {crit }}$, are spatially uniform (or piecewise constant). We set the standard value for $\phi_{r}=7.33 \times 10^{-2}$ and consider the subcases (i) $f_{\text {crit }}=3 \times 10^{-5}$ for $0 \leq \theta \leq 1$ (Fig. 4 A), and (ii) $f_{\text {crit }}=0.3$ for $\theta>0.13$ while $f_{\text {crit }}=3 \times 10^{-5}$ for $\theta \leq 0.13$ (Fig. 4B), as were explored in Roberts (2022). These subcases correspond to the situation in which the TF threshold concentration lies beneath the minimum healthy TF value at all retinal locations (i), and the situation in which foveal cones are afforded special protection compared to the rest of the retina, such that they can withstand lower TF concentrations (ii). For notational simplicity, we shall refer to subcase (ii) simply as $f_{\text {crit }}=0.3$ in what follows. As with Figs. 6 9, we consider both Scaling 1 and Scaling 2 (see Inverse Problem) on the rate of TF production by rods, $\alpha$, and the rate of TF consumption by cones, $\beta$, calculating both analytical and numerical solutions.

Cone degeneration initiates at the fovea $(\theta=0)$ in Fig. $4 \mathrm{~A}$ and at $\theta=0.13$ in Fig. 4 $\mathrm{B}$, spreading peripherally (rightwards) in both cases, while degeneration also initiates at the ora serrata $(\theta=1)$ under Scaling 2 in both Fig. 4A and Fig. 4B, spreading centrally. Degeneration occurs earlier in Fig. 4B than in Fig. 4A and earlier for Scaling 2 than for Scaling 1 (except near the fovea in Fig. 4A). Numerical and analytical solutions agree well, only diverging close to fovea in Fig. 4A, where the analytical solution breaks down. None of these patterns of degeneration match those seen in vivo (see Fig. 2).

In Figs. 6- 9 we calculate the $\phi_{r}(\theta)=\phi_{r_{\text {inv }}}(\theta)$ and $f_{\text {crit }}(\theta)=f_{\text {crit }}{ }_{\text {inv }}(\theta)$ profiles required to qualitatively replicate the cone degeneration profiles, $t_{\text {degen }}(\theta)$, observed in vivo (Fig. 5), by solving the associated inverse problems (see Inverse Problem). As noted in the Inverse Problem section, when searching for $\phi_{r_{\text {inv }}}(\theta)$, we hold the TF threshold concentration constant at $f_{\text {crit }}(\theta)=f_{\text {crit }}=3 \times 10^{-5}$, while, when searching for $f_{\text {crit }_{\text {inv }}}(\theta)$, we hold the rate of mutation-induced rod loss constant at $\phi_{r}(\theta)=\phi_{r}=7.33 \times$ $10^{-2}$. Analytical inverses are plotted across the domain $(0 \leq \theta \leq 1)$, while numerical inverses are calculated and plotted only at those locations (eccentricities) where the analytical inverse fails to generate a $t_{\text {degen }}(\theta)$ profile matching the target profile (as determined by visual inspection, the $t_{\text {degen }}(\theta)$ and target profiles being visually indistinguishable outside of these regions).

In Fig. 6 we calculate inverses for a Uniform degeneration profile. While this pattern is not typically observed in humans (though present in animal models of RP), we consider this case as a point of comparison with the non-uniform patterns explored in Figs. $7 f 9$. Both inverses, $\phi_{r_{\text {inv }}}(\theta)$ and $f_{\text {crit }_{\text {inv }}}(\theta)$, are monotone increasing for Scaling 1, and increase initially for Scaling 2 before reaching a maximum and decreasing toward the ora serrata (at $\theta=1$ ). Consequently, Scaling 1 and 2 inverses, while close near the fovea $(\theta=0)$, diverge toward the ora serrata, this effect being more prominent for $f_{\text {crit }}(\theta)$. The inverse profiles have a similar shape to the $t_{\text {degen }}(\theta)$ profiles in Fig. 4 (see Discussion). Numerical solutions reveal lower values of the inverses near the fovea, where the analytical approximations break down.

Inverses for linear (Fig. 7A and B), concave up (quadratic) (Fig. 7C and D) and concave down (quadratic) (Fig. 7E and F) Pattern 1A degeneration profiles are shown in Fig. 7. Inverses are monotone increasing functions for both Scalings 1 and 2 in Fig. 7A,B,E and F, and for Scaling 1 in Fig. 7C and D, while the 
bioRxiv preprint doi: https://doi.org/10.1101/2021.09.04.458968; this version posted February 14, 2022. The copyright holder for this preprint (which was not certified by peer review) is the author/funder, who has granted bioRxiv a license to display the preprint in perpetuity. It is made available under aCC-BY-NC-ND 4.0 International license.

\section{DISCUSSION} that region.

inverses increase initially for Scaling 2 before reaching a maximum and decreasing toward the ora serrata in Fig. $7 \mathrm{C}$ and D. Numerical solutions reveal lower values of the inverses near the fovea, where the analytical approximations break down.

Fig. 8 shows inverses for linear (Fig. 8A and B), quadratic (Fig. 8C and D) and exponential (Fig. $8 \mathrm{E}$ and F) Pattern 1B degeneration profiles. Inverses resemble vertically flipped versions of the $t_{\text {degen }}(\theta)$ profiles in Fig. 5 C (see Discussion). Numerical solutions reveal lower values of the inverses near the fovea, where the analytical approximations break down, and higher values in some regions away from the fovea in Fig. 8A-D. The discontinuities in the linear and quadratic cases are biologically unrealistic, though consistent with the idealised qualitative target cone degeneration patterns in Fig. $5 \mathrm{C}$.

In Fig. 9 we calculate inverses for linear 1 (Fig. 9A and B), linear 2 (Fig. 9C and D), quadratic (Fig. 9E and F) and cubic (Fig. $9 \mathrm{G}$ and $\mathrm{H}$ ) Pattern 3 degeneration profiles. Inverses resemble vertically flipped versions of the $t_{\text {degen }}(\theta)$ profiles in Fig. 5D (see Discussion). Numerical solutions reveal lower values of the inverses near the fovea, where the analytical approximations break down, and higher values in some regions away from the fovea in Fig. $9 \mathrm{C}-\mathrm{F}$ and $\mathrm{H}$. Similarly to Fig. 8 , the discontinuities in the linear 2 and quadratic cases are biologically unrealistic, though consistent with the idealised qualitative target cone degeneration patterns in Fig. 5D.

Lastly, in Fig. 10, we show simulation results of proportional cone loss for analytical and numerical $\phi_{r_{\text {inv }}}(\theta)$ and $f_{\text {critinv }_{\text {inv }}}(\theta)$, for Uniform (Scaling 1, Fig. 10A-D), concave up Pattern 1A (Scaling 1, Fig. 10E-H), linear Pattern 1B (Scaling 2, Fig. 10[-L) and quadratic Pattern 3 (Scaling 2, Fig. 10M-P) target degeneration profiles. Cone degeneration profiles generally show good agreement with the target $t_{\text {degen }}(\theta)$ profiles. There is some divergence from $t_{\text {degen }}(\theta)$ for the analytical inverses near the fovea and at discontinuous or nonsmooth portions of $t_{\text {degen }}(\theta)$; this is mostly corrected by the numerical inverses. This correction is not perfect near the centre of the fovea, where cones still degenerate earlier than the target profiles. This occurs because it is necessary to replace the Heaviside step function in $\lambda_{2}(f)$ (see Eqn. (3)) with a hyperbolic tanh function to satisfy the smoothness requirements for the numerical solver, with the result that the initiation of cone degeneration is sensitive to the low TF concentrations $\left(f(\theta, t)<10^{-4}\right)$ in

The spatio-temporal patterns of retinal degeneration observed in human retinitis pigmentosa (RP) are well characterised; however, the mechanistic explanation for these patterns has yet to be conclusively determined. In this paper, we have formulated a one-dimensional (1D) reaction-diffusion partial differential equation (PDE) model (modified from Roberts, 2022) to predict RP progression under the trophic factor (TF) hypothesis. Using this model, we solved inverse problems to determine the rate of mutation-induced rod loss profiles, $\phi_{r}(\theta)=\phi_{r_{\text {inv }}}(\theta)$, and TF threshold concentration profiles, $f_{\text {crit }}(\theta)=f_{\text {crit }}(\theta)$, that would be required to generate spatio-temporal patterns of cone degeneration qualitatively resembling those observed in vivo, were the TF mechanism solely responsible for RP progression. In reality, multiple mechanisms (including oxidative damage and metabolic dysregulation, Punzo et al., 2009, 2012; Stone et al., 1999; (Travis et al., 1991; Valter et al., 1998) likely operate in tandem to drive the initiation and propagation of retinal degeneration in RP. By using mathematics to isolate the TF mechanism, in a way that would be impossible to achieve experimentally, we are able to determine the conditions under which the TF mechanism alone would recapitulate known phenotypes. Having identified these conditions, this paves the way for future biomedical and experimental studies to test our predictions. 
bioRxiv preprint doi: https://doi.org/10.1101/2021.09.04.458968; this version posted February 14, 2022. The copyright holder for this preprint (which was not certified by peer review) is the author/funder, who has granted bioRxiv a license to display the preprint in perpetuity. It is made available under aCC-BY-NC-ND 4.0 International license.

Other mechanisms may give rise to spatio-temporal patterns of retinal degeneration different from those predicted for the TF mechanism and may do so using fewer assumptions. For example, our previous work on oxygen toxicity in RP demonstrated that this mechanism can replicate visual field loss Pattern 1 (especially 1B) and the late far-peripheral degeneration stage of Pattern 3, without imposing heterogeneities on the rod decay rate or photoreceptor susceptibility to oxygen toxicity (Roberts et al., 2017, 2018). Further, we hypothesise that the toxic substance hypothesis (in which dying rods release a chemical which kills neighbouring photoreceptors) is best able to explain the early mid-peripheral loss of photoreceptors in Patterns 2 and 3, given the high density of rods in this region. In future work, we will explore the toxic substance and other hypotheses, ultimately combining them together in a more comprehensive modelling framework, aimed at explaining and predicting all patterns of retinal degeneration in RP.

Spatially uniform $\phi_{r}(\theta)$ and $f_{\text {crit }}(\theta)$ profiles fail to replicate any of the in vivo patterns of degeneration (Fig. 4), showing that heterogenous profiles are required, all else being equal. Throughout this paper we have considered two scalings on the rate of TF production by rods, $\alpha$, and the rate of TF consumption by cones, $\beta$ (denoted as Scalings 1 and 2, see the Inverse Problem section for details). Under Scaling 1, the rod:cone ratio (Fig. $3 \mathrm{~B}$ ) dominates the model behaviour (see Eqn. (6)), leading to a monotone, central to peripheral pattern of degeneration, while under Scaling 2, the trophic factor decay term, $\eta f$, enters the dominant balance (see Eqn. (9)), such that degeneration initiates at both the fovea and (later) at the ora serrata, the degenerative fronts meeting in the mid-/far-periphery (Fig. 4).

As discussed in the Inverse Problem section, the rate of mutation-induced rod loss, $\phi_{r}(\theta)$, is known to be spatially heterogeneous (Curcio et al., 1993). However, to the best of our knowledge, we are the first to suggest that the intrinsic susceptibility of cones to RdCVF deprivation, characterised in our models by the TF threshold concentration, $f_{\text {crit }}(\theta)$, may vary across the retina. Assuming it does vary, what might account for this phenomenon? There is a precedent for special protection being provided to localised parts of the retina. For example, experiments in mice have found that production of basic fibroblast growth factor (bFGF) and glial fibrillary acidic protein (GFAP) is permanently upregulated along the retinal edges, at the ora serrata and optic disc, to protect against elevated stress in these regions (Mervin and Stone, 2002; Stone et al., 2005). By analogy, we speculate that, in the human retina, protective factors may be upregulated at the fovea to compensate for the low RdCVF concentrations in that region, lowering the local value of $f_{\text {crit }}(\theta)$. This hypothesis awaits experimental confirmation.

We solved the inverse functions, $\phi_{r_{\text {inv }}}(\theta)$ and $f_{\text {critinv }_{\text {inv }}}(\theta)$, both analytically (algebraically) and numerically (computationally). Analytical solutions are approximations; however, they have the advantage of being easier to compute (increasing their utility for biomedical researchers) and provide a more intuitive understanding of model behaviour, while numerical solutions are more accurate, though computationally expensive. We calculated the inverses for a range of target cone degeneration profiles, consisting of a Uniform profile and profiles which qualitatively replicate those found in vivo: Pattern 1A, Pattern 1B and Pattern 3 (Pattern 2 being inaccessible to a 1D model; see Table 3 and Fig. 57.

The shapes of the inverse functions are determined partly by the rod and cone distributions, $\tilde{p}_{r}(\theta)$ and $\tilde{p}_{c}(\theta)$, and partly by the target cone degeneration profile, $t_{\text {degen }}(\theta)$ (see Eqs. (7), (8), (10) and (11)). As such, in the Uniform case (Fig. 6), the Scaling 1 inverse profiles take a similar shape to the rod:cone ratio (Fig. 3B), the inverses being lower towards the fovea to compensate for the smaller rod:cone ratio and hence lower supply of TF to each cone. The Scaling 2 inverse profiles follow a similar trend but decrease toward the ora serrata after peaking in the mid-/far-periphery due to the greater influence of the trophic factor decay term under this scaling. Interestingly, the shapes of these inverse profiles bear a striking resemblance to the cone degeneration profiles for spatially uniform $\phi_{r}(\theta)$ and $f_{\text {crit }}(\theta)$ (Fig. 4). This is because lower 
bioRxiv preprint doi: https://doi.org/10.1101/2021.09.04.458968; this version posted February 14, 2022. The copyright holder for this preprint (which was not certified by peer review) is the author/funder, who has granted bioRxiv a license to display the preprint in perpetuity. It is made available under aCC-BY-NC-ND 4.0 International license.

370 values of the inverses are required to delay degeneration, in those regions where cones would otherwise degenerate earlier, to achieve a uniform degeneration profile. The inverse functions resemble vertically flipped versions of the target cone degeneration profiles for Patterns 1A, 1B and 3 (Figs. 7f 9), this being more apparent for Patterns 1B and 3 due to their more distinctive shapes. This makes sense since lower inverse values are required for later degeneration times. Scaling 2 inverses typically lie below Scaling 1 inverses, compensating for the fact that degeneration generally occurs earlier under Scaling 2 than under Scaling 1 for any given $\phi_{r}(\theta)$ and $f_{\text {crit }}(\theta)$.

Analytical inverses give rise to cone degeneration profiles that accurately match the target cone degeneration profiles, except near the fovea (centred at $\theta=0$, where the validity of the analytical approximation breaks down) and where the target $t_{\text {degen }}(\theta)$ profile is nonsmooth or discontinuous (i.e. linear and quadratic Pattern 1B, and linear 1, linear 2 and quadratic Pattern 3; see Fig. 10 for examples). Numerical inverses improve accuracy in these regions, consistently taking lower values near the fovea, delaying degeneration where it occurs prematurely under the analytical approximation.

We have assumed throughout this study that at least one of $\phi_{r}(\theta)$ and $f_{\text {crit }}(\theta)$ is spatially uniform. It is possible, however, that both vary spatially. In this case there are no unique inverses; however, if the profile for one of these functions could be measured experimentally, then the inverse problem for the remaining function could be solved as in this paper.

This work could be extended both experimentally and theoretically. Experimental and biomedical studies could measure how the rate of mutation-induced rod loss and TF threshold concentration vary with location in the retina, noting the spatio-temporal pattern of retinal degeneration and comparing with the inverse $\phi_{r_{\text {inv }}}(\theta)$ and $f_{\text {critinv }}(\theta)$ profiles predicted by our models. Curcio et al. (1993) have previously measured variation in the rate of rod loss in normal (non-RP) human retinas (where rods degenerated most rapidly in the central retina); however, to the best of our knowledge, detailed measurements have not been made in the RP retina. The parameter $f_{\text {crit }}$ is less straightforward to measure. Léveillard et al. (2004) incubated coneenriched primary cultures from chicken embryos with glutathione S-transferase-RdCVF (GST-RdCVF) fusion proteins, doubling the number of living cells per plate compared with GST alone. If experiments of this type could be repeated for a range of controlled RdCVF concentrations, then the value of $f_{\text {crit }}$ could be identified. Determining the spatial variation of $f_{\text {crit }}(\theta)$ in a foveated human-like retina may not be possible presently; however, the recent development of retinal organoids provides promising steps in this direction (Fathi et al., 2021; O'Hara-Wright and Gonzalez-Cordero, 2020). If organoids could be developed with a specialised macular region, mirroring that found in vivo, then the minimum RdCVF concentration required to maintain cones in health could theoretically be tested at a variety of locations. Further, the distribution of RdCVF, predicted in our models, could theoretically be measured in post-mortem human eyes using fluorescent immunohistochemistry, as was done for the protein neuroglobin by Ostojić et al. (2008) and Rajendram and Rao (2007). In particular, it would be interesting to see if RdCVF concentration varies with retinal eccentricity as starkly as our model predicts, with extremely low levels in the fovea.

In future work, we will extend our mathematical model to two spatial dimensions, accounting for variation in the azimuthal/circumferential dimension (allowing us to capture radially asymmetric aspects of visual field loss Patterns 2 and 3, and to account for azimuthal variation in the rod and cone distributions), and use quantitative target cone degeneration patterns derived from SD-OCT imaging of RP patients (e.g. as in Escher et al., 2012). We will also adapt the model to consider animal retinas for which the photoreceptor distribution has been well characterised (e.g. rats, mice and pigs, Chandler et al., 1999; Gaillard et al., 2009: Ortín-Martínez et al., 2014). 
In conclusion, we have formulated and solved a mathematical inverse problem to determine the rate of mutation-induced rod loss and TF threshold concentration profiles required to explain the spatio-temporal patterns of retinal degeneration observed in human RP. Inverse profiles were calculated for a set of qualitatively distinct degeneration patterns, achieving a close match with the target cone degeneration profiles. Predicted inverse profiles await future experimental verification.

\section{CONFLICT OF INTEREST STATEMENT}

The author declares that the research was conducted in the absence of any commercial or financial relationships that could be construed as a potential conflict of interest.

\section{AUTHOR CONTRIBUTIONS}

PAR: conceptualisation, methodology, software, validation, formal analysis, investigation, data curation, writing - original draft, writing —- review and editing, visualisation, and project administration.

\section{FUNDING}

P.A.R. is funded by BBSRC (BB/R014817/1).

\section{ACKNOWLEDGMENTS}

P.A.R. thanks Tom Baden for allowing the time to pursue this research. P.A.R. also thanks the reviewers for their helpful and insightful comments.

\section{REFERENCES}

Aït-Ali, N., Fridlich, R., Millet-Puel, G., Clérin, E., Delalande, F., Jaillard, C., et al. (2015). Rod-derived cone viability factor promotes cone survival by stimulating aerobic glycolysis. Cell 161, 817-832. doi:https://doi.org/10.1016/j.cell.2015.03.023

Burns, J., Clarke, G., and Lumsden, C. J. (2002). Photoreceptor death: Spatiotemporal patterns arising from one-hit death kinetics and a diffusible cell death factor. Bull. Math. Biol. 64, 1117-1145. doi:https://doi.org/10.1006/bulm.2002.0320

Camacho, E. T., Brager, D., Elachouri, G., Korneyeva, T., Millet-Puel, G., Sahel, J. A., et al. (2019). A mathematical analysis of aerobic glycolysis triggered by glucose uptake in cones. Sci. Rep. 9, 4162. doi:https://doi.org/10.1038/s41598-019-39901-z

Camacho, E. T., Colón Vélez, M. A., Hernández, D. J., Bernier, U. R., van Laarhoven, J., and Wirkus, S. (2010). A mathematical model for photoreceptor interactions. J. Theor. Biol. 267, 638-646. doi:https://doi.org/10.1016/j.jtbi.2010.09.006

Camacho, E. T., Dobreva, A., Larripa, K., Rădulescu, A., Schmidt, D., and Trejo, I. (2021). Mathematical Modeling of Retinal Degeneration: Aerobic Glycolysis in a Single Cone (Cham: Springer International Publishing), vol. 22 of Association for Women in Mathematics Series. 135-178. doi:https://doi.org/10. 1007/978-3-030-57129-0_7

Camacho, E. T., Lenhart, S., Melara, L. A., Villalobos, M. C., and Wirkus, S. (2020). Optimal control with MANF treatment of photoreceptor degeneration. Math. Med. Biol. 37, 1-21. doi:https://doi.org/10.1093/ imammb/dqz003

Camacho, E. T., Léveillard, T., Sahel, J. A., and Wirkus, S. (2016a). Mathematical model of the role of RdCVF in the coexistence of rods and cones in a healthy eye. Bull. Math. Biol. 78, 1394-1409. doi:https://doi.org/10.1007/s11538-016-0185-x 
bioRxiv preprint doi: https://doi.org/10.1101/2021.09.04.458968; this version posted February 14, 2022. The copyright holder for this preprint (which was not certified by peer review) is the author/funder, who has granted bioRxiv a license to display the preprint in perpetuity. It is made available under aCC-BY-NC-ND 4.0 International license.

447 Camacho, E. T., Melara, L. A., Villalobos, M. C., and Wirkus, S. (2014). Optimal control in the treatment of retinitis pigmentosa. Bull. Math. Biol. 76, 292-313. doi:https://doi.org/10.1007/s11538-013-9919-1

Camacho, E. T., Punzo, C., and Wirkus, S. A. (2016b). Quantifying the metabolic contribution to photoreceptor death in retinitis pigmentosa via a mathematical model. J. Theor. Biol. 408, 75-87. doi:https://doi.org/10.1016/j.jtbi.2016.08.001

Camacho, E. T., Radulescu, A., and Wirkus, S. (2016c). Bifurcation analysis of a photoreceptor interaction model for retinitis pigmentosa. Commun. Nonlinear Sci. Numer. Simulat. 38, 267-276. doi:https: //doi.org/10.1016/j.cnsns.2016.02.030

Camacho, E. T. and Wirkus, S. (2013). Tracing the progression of retinitis pigmentosa via photoreceptor interactions. J. Theor. Biol. 317, 105-118. doi:https://doi.org/10.1016/j.jtbi.2012.09.034

Chandler, M. J., Smith, P. J., Samuelson, D. A., and Mackay, E. O. (1999). Photoreceptor density of the domestic pig retina. Vet. Ophthalmol. 2, 179-184. doi:https://doi.org/10.1046/j.1463-5224.1999.00077.x

Colón Vélez, M. A., Hernández, D. J., Bernier, U. R., van Laarhoven, J., and Camacho, E. T. (2003). Mathematical Models for Photoreceptor Interactions. Tech. rep., Cornell University, Department of Biological Statistics and Computational Biology

Curcio, C. A., Millican, C. L., Allen, K. A., and Kalina, R. E. (1993). Aging of the human photoreceptor mosaic: evidence for selective vulnerability of rods in central retina. Invest. Ophthalmol. Vis. Sci. 34, 3278-3296

Curcio, C. A., Sloan, K. R., Kalina, R. E., and Hendrickson, A. E. (1990). Human photoreceptor topography. J. Comp. Neurol. 292, 497-523. doi:https://doi.org/10.1002/cne.902920402

Daiger, S. P., Bowne, S. J., and Sullivan, L. S. (2007). Perspective on genes and mutations causing retinitis pigmentosa. Arch. Ophthalmol. 125, 151-158. doi:10.1001/archopht.125.2.151

Eden, E., Geva-Zatorsky, N., Issaeva, I., Cohen, A., Dekel, E., Danon, T., et al. (2011). Proteome half-life dynamics in living human cells. Science 331, 764-768. doi:10.1126/science.1199784

Escher, P., Tran, H. V., Vaclavik, V., Borruat, F. X., Schorderet, D. F., and Munier, F. L. (2012). Double concentric autofluorescence ring in NR2E3-p.G56R-linked autosomal dominant retinitis pigmentosa. Invest. Ophthalmol. Vis. Sci. 53, 4754-4764

Fathi, M., Ross, C. T., and Hosseinzadeh, Z. (2021). Functional 3-dimensional retinal organoids: Technological progress and existing challenges. Front. Neurosci. 15, 668857. doi:https://doi.org/10.3389/fnins. 2021.668857

Fintz, A. C., Audo, I., Hicks, D., Mohand-Saïd, S., Léveillard, T., and Sahel, J. (2003). Partial characterization of retina-derived cone neuroprotection in two culture models of photoreceptor degeneration. Invest. Ophthalmol. Vis. Sci. 44, 818-825. doi:https://doi.org/10.1167/iovs.01-1144

Gaillard, F., Kuny, S., and Sauvé, Y. (2009). Topographic arrangement of S-cone photoreceptors in the retina of the diurnal nile grass rat (arvicanthis niloticus). Invest. Ophthalmol. Vis. Sci. 50, 5426-5434. doi:https://doi.org/10.1167/iovs.09-3896

Grover, S., Fishman, G. A., and Brown Jr, J. (1998). Patterns of visual field progression in patients with retinitis pigmentosa. Ophthalmology 105, 1069-1075. doi:https://doi.org/10.1016/S0161-6420(98) 96009-2

Gupta, N., Brown, K. E., and Milam, A. H. (2003). Activated microglia in human retinitis pigmentosa, late-onset retinal degeneration, and age-related macular degeneration. Exp. Eye Res. 76, 463-471. doi:https://doi.org/10.1016/S0014-4835(02)00332-9

Hamel, C. (2006). Retinitis pigmentosa. Orphanet. J. Rare Dis. 1, 40. doi:https://doi.org/10.1186/ 1750-1172-1-40 
bioRxiv preprint doi: https://doi.org/10.1101/2021.09.04.458968; this version posted February 14, 2022. The copyright holder for this preprint (which was not certified by peer review) is the author/funder, who has granted bioRxiv a license to display the preprint in perpetuity. It is made available under aCC-BY-NC-ND 4.0 International license.

Hartong, D. T., Berson, E. L., and Dryja, T. P. (2006). Retinitis pigmentosa. Lancet 368, 1795-1809. doi:https://doi.org/10.1016/S0140-6736(06)69740-7

Léveillard, T., Mohand-Saïd, S., Lorentz, O., Hicks, D., Fintz, A. C., Clérin, E., et al. (2004). Identification and characterization of rod-derived cone viability factor. Nat. Genet. 36, 755-759. doi:https://doi.org/10. $1038 / n g 1386$

Léveillard, T. and Sahel, J. A. (2017). Metabolic and redox signaling in the retina. Cell. Mol. Life Sci. 74, 3649-3665. doi:https://doi.org/10.1007/s00018-016-2318-7

Mei, X., Chaffiol, A., Kole, C., Yang, Y., Millet-Puel, G., Clérin, E., et al. (2016). The thioredoxin encoded by the rod-derived cone viability factor gene protects cone photoreceptors against oxidative stress. Antioxidants \& Redox Signaling 24, 909-923. doi:https://doi.org/10.1089/ars.2015.6509

Mervin, K. and Stone, J. (2002). Developmental death of photoreceptors in the C57BL/6J mouse: Association with retinal function and self-protection. Exp. Eye Res. 75, 703-713. doi:https://doi.org/10. 1006/exer.2002.2063

Mohand-Saïd, S., Deudon-Combe, A., Hicks, D., Simonutti, M., Forster, V., Fintz, A. C., et al. (1998). Normal retina releases a diffusible factor stimulating cone survival in the retinal degeneration mouse. Proc. Natl. Acad. Sci. 95, 8357-8362. doi:https://doi.org/10.1073/pnas.95.14.8357

Mohand-Saïd, S., Hicks, D., Dreyfus, H., and Sahel, J. A. (2000). Selective transplantation of rods delays cone loss in a retinitis pigmentosa model. Arch. Ophthalmol. 118, 807-811. doi:10.1001/archopht.118.6. 807

Mohand-Saïd, S., Hicks, D., Simonutti, M., Tran-Minh, D., Deudon-Combe, A., Dreyfus, H., et al. (1997). Photoreceptor transplants increase host cone survival in the retinal degeneration (rd) mouse. Ophthalmic Res. 29, 290-297. doi:https://doi.org/10.1159/000268027

Newton, F. and Megaw, R. (2020). Mechanisms of photoreceptor death in retinitis pigmentosa. Genes 11, 1120. doi:https://doi.org/10.3390/genes 11101120

O'Hara-Wright, M. and Gonzalez-Cordero, A. (2020). Retinal organoids: a window into human retinal development. Development 147, dev189746. doi:https://doi.org/10.1242/dev.189746

Ortín-Martínez, A., Nadal-Nicolás, F. M., Jiménez-López, M., Alburquerque-Béjar, J. J., Nieto-López, L., García-Ayuso, D., et al. (2014). Number and distribution of mouse retinal cone photoreceptors: Differences between an albino (Swiss) and a pigmented (C57/BL6) strain. PLoS One 9, e102392. doi:https://doi.org/10.1371/journal.pone.0102392

Ostojić, J., Grozdanić, S. D., Syed, N. A., Hargrove, M. S., Trent, J. T., Kuehn, M. H., et al. (2008). Patterns of distribution of oxygen-binding globins, neuroglobin and cytoglobin in human retina. Arch. Ophthalmol. 126, 1530-1536. doi:10.1001/archopht.126.11.1530

Oyster, C. W. (1999). The Human Eye: Structure and Function (Sinauer Associates Inc.)

Punzo, C., Kornacker, K., and Cepko, C. L. (2009). Stimulation of the insulin/mTOR pathway delays cone death in a mouse model of retinitis pigmentosa. Nat. Neurosci. 12, 44-52. doi:https://doi.org/10.1038/ nn. 2234

Punzo, C., Xiong, W., and Cepko, C. L. (2012). Loss of daylight vision in retinal degeneration: Are oxidative stress and metabolic dysregulation to blame? J. Biol. Chem. 287, 1642-1648. doi:https: //doi.org/10.1074/jbc.R111.304428

Rajendram, R. and Rao, N. A. (2007). Neuroglobin in normal retina and retina from eyes with advanced glaucoma. Br. J. Ophthalmol. 91, 663-666. doi:http://dx.doi.org/10.1136/bjo.2006.093930

Ripps, H. (2002). Cell death in retinitis pigmentosa: Gap junctions and the 'bystander' effect. Exp. Eye Res. 74, 327-336. doi:https://doi.org/10.1006/exer.2002.1155 
Roberts, P. A. (2022). Mathematical models of retinitis pigmentosa: The trophic factor hypothesis. J. Theor. Biol. 534, 110938. doi:https://doi.org/10.1016/j.jtbi.2021.110938

Roberts, P. A., Gaffney, E. A., Luthert, P. J., Foss, A. J. E., and Byrne, H. M. (2016a). Mathematical and computational models of the retina in health, development and disease. Prog. Retin. Eye. Res. 53, 48-69. doi:https://doi.org/10.1016/j.preteyeres.2016.04.001

Roberts, P. A., Gaffney, E. A., Luthert, P. J., Foss, A. J. E., and Byrne, H. M. (2016b). Retinal oxygen distribution and the role of neuroglobin. J. Math. Biol. 73, 1-38. doi:https://doi.org/10.1007/ s00285-015-0931-y

Roberts, P. A., Gaffney, E. A., Luthert, P. J., Foss, A. J. E., and Byrne, H. M. (2017). Mathematical models of retinitis pigmentosa: The oxygen toxicity hypothesis. J. Theor. Biol. 425, 53-71. doi:https: //doi.org/10.1016/j.jtbi.2017.05.006

Roberts, P. A., Gaffney, E. A., Whiteley, J. P., Luthert, P. J., Foss, A. J. E., and Byrne, H. M. (2018). Predictive mathematical models for the spread and treatment of hyperoxia-induced photoreceptor degeneration in retinitis pigmentosa. Invest. Ophthalmol. Vis. Sci. 59, 1238-1249. doi:https://doi.org/10. 1167/iovs.17-23177

Stone, J., Maslim, J., Valter-Kocsi, K., Mervin, K., Bowers, F., Chu, Y., et al. (1999). Mechanisms of photoreceptor death and survival in mammalian retina. Prog. Retin. Eye Res. 18(6), 689-735. doi:https://doi.org/10.1016/S1350-9462(98)00032-9

Stone, J., Mervin, K., Walsh, N., Valter, K., Provis, J. M., and Penfold, P. L. (2005). Photoreceptor stability and degeneration in mammalian retina: Lessons from the edge. In Macular Degeneration, eds. P. Penfold and J. Provis (Springer Berlin Heidelberg). 149-165. doi:http://dx.doi.org/10.1007/3-540-26977-0_9

Travis, G. H., Sutcliffe, J. G., and Bok, D. (1991). The retinal degeneration slow (rds) gene product is a photoreceptor disc membrane-associated glycoprotein. Neuron 6, 61-70. doi:https://doi.org/10.1016/ 0896-6273(91)90122-G

Valter, K., Maslim, J., Bowers, F., and Stone, J. (1998). Photoreceptor dystrophy in the RCS rat: roles of oxygen, debris, and bFGF. Invest. Ophthalmol. Vis. Sci. 39, 2427-2442

Wifvat, K., Camacho, E. T., Wirkus, S., and Léveillard, T. (2021). The role of RdCVFL in a mathematical model of photoreceptor interactions. J. Theor. Biol. 520, 110642. doi:https://doi.org/10.1016/j.jtbi.2021. 110642

Yang, Y., Mohand-Said, S., Danan, A., Simonutti, M., Fontaine, V., Clérin, E., et al. (2009). Functional cone rescue by RdCVF protein in a dominant model of retinitis pigmentosa. Mol. Ther. 17, 787-795. doi:https://doi.org/10.1038/mt.2009.28 

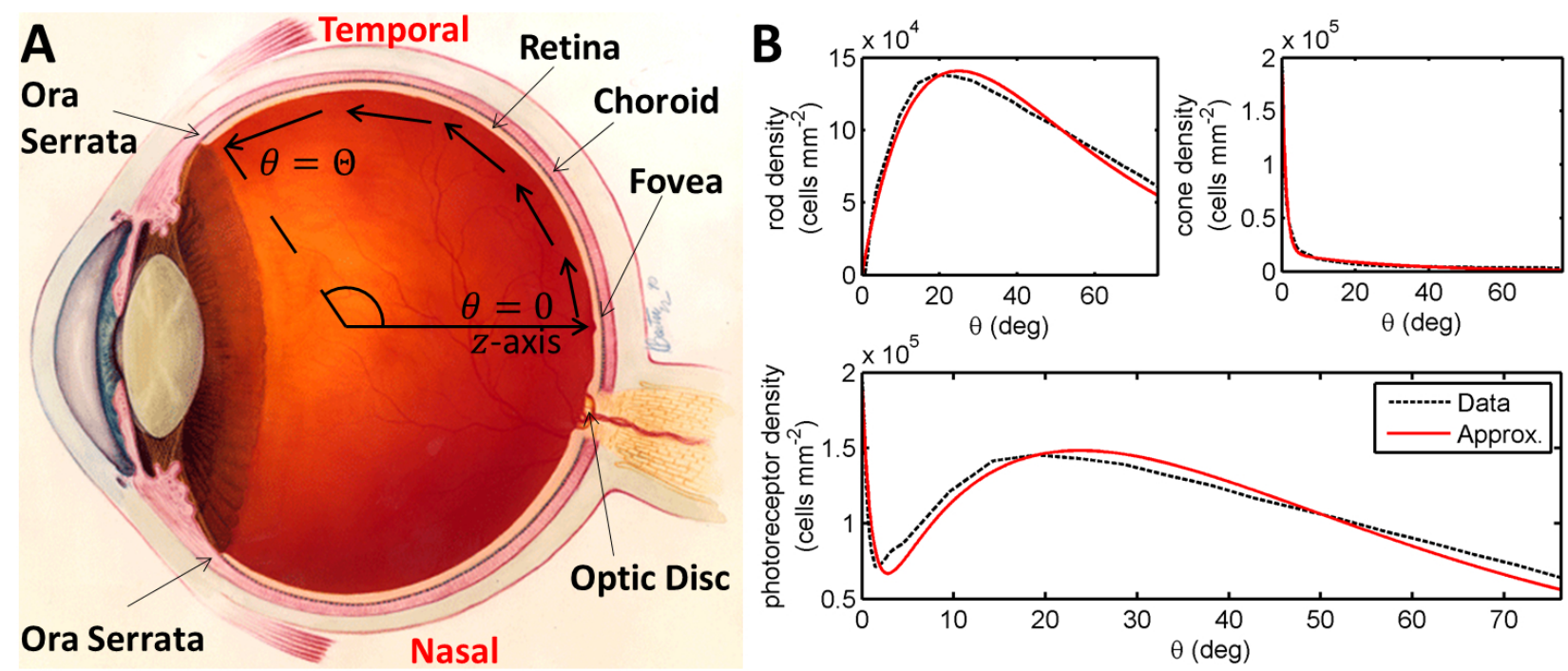

Figure 1. Diagrams of the human eye and retinal photoreceptor distribution (reproduced, with permission, from Roberts et al., 2017). (A) Diagram of the (right) human eye, viewed in the transverse plane, illustrating the mathematical model geometry. The model is posed on a domain spanning the region between the foveal centre, at $\theta=0$, and the ora serrata, at $\theta=\Theta$, along the temporal horizontal meridian, where $\theta$ measures the eccentricity. Figure originally reproduced, with modifications, from http://www.nei.nih.gov/health/coloboma/ coloboma. asp, courtesy: National Eye Institute, National Institutes of Health (NEI/NIH). (B) Measured and fitted photoreceptor profiles, along the temporal horizontal meridian, in the human retina. Cone profile: $\tilde{p}_{c}(\theta)=$ $B_{1} e^{-b_{1} \theta}+B_{2} e^{-b_{2} \theta}$, and rod profile: $\tilde{p}_{r}(\theta)=B_{3} \theta e^{-b_{3} \theta}$ (see Table 2 for dimensionless parameter values). The photoreceptor profile is the sum of the rod and cone profiles $\left(\tilde{p}_{r}(\theta)+\tilde{p}_{c}(\theta)\right)$. Experimental data provided by Curcio and published in Curcio et al. (1990).

\section{FIGURES \& TABLES}


bioRxiv preprint doi: https://doi org/10.1101/2021.09 04.458968; this version posted February 14, 2022. The copyright holder for this preprint (which was not certified by peer review) is the author/funder, who has granted bioRxiv a license to display the preprint in perpetuity. It is made available under aCC-BY-NC-ND 4.0 International license.



Figure 2. Characteristic patterns of visual field loss in human RP (reproduced, with permission, from Roberts et al., 2018). Visual field loss patterns can be classified into three cases and six subcases (classification system described in Grover et al., 1998). Large grey arrows indicate transitions between stages of visual field loss and small red arrows indicate the direction of scotoma (blind spot) propagation. See text for details.

Table 1. Variables employed in the non-dimensional mathematical model (Eqs. (1)-(5)).

\begin{tabular}{ll}
\hline Variable & Description \\
\hline$\theta$ & Eccentricity \\
$t$ & Time \\
$f(\theta, t)$ & Trophic factor concentration \\
$p_{r}(\theta, t)$ & Rod density \\
$p_{c}(\theta, t)$ & Cone density \\
\hline
\end{tabular}



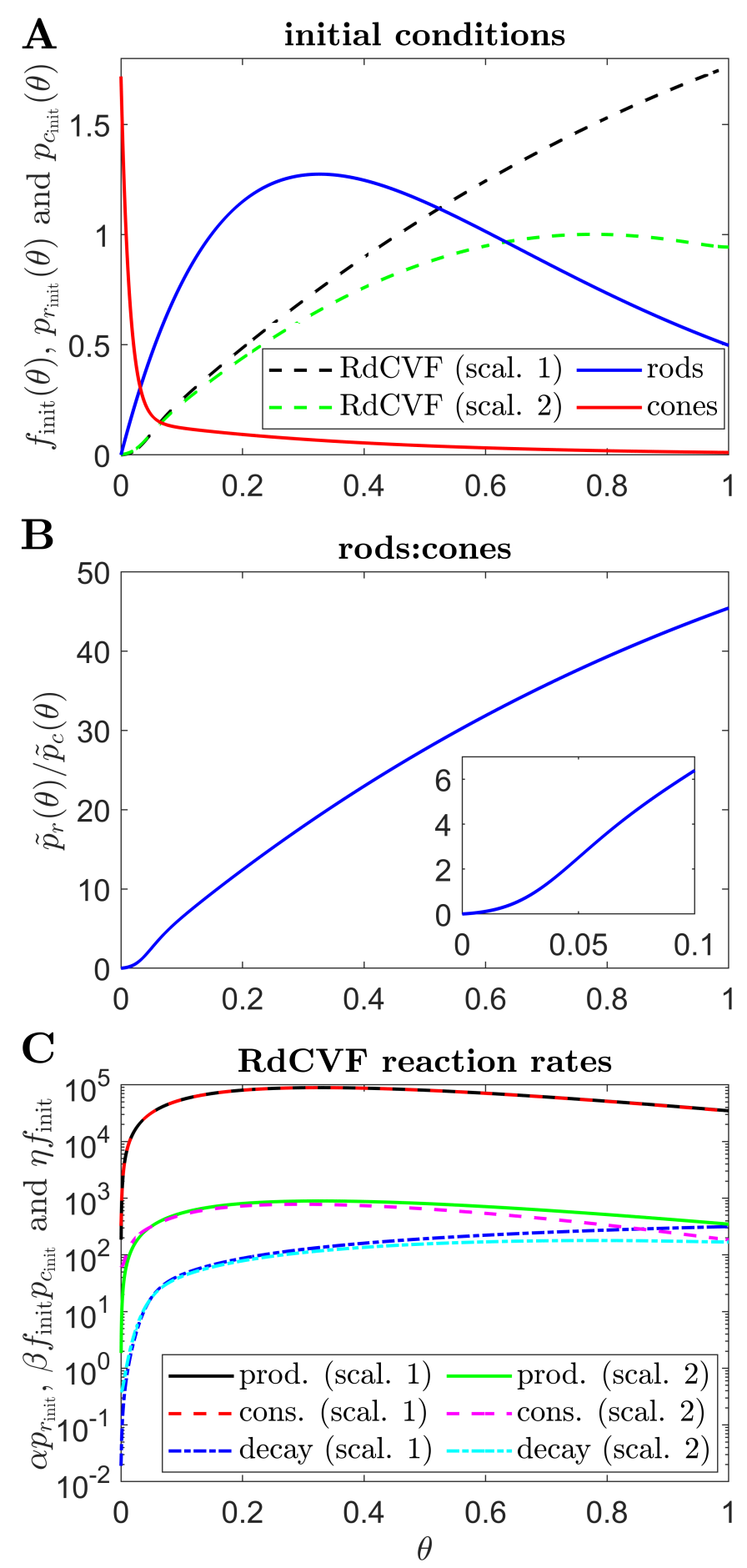

Figure 3. Initial conditions, ratio of rods to cones and RdCVF reaction rates. (A) initial conditions used in all simulations, consisting of healthy rod and cone profiles and the corresponding RdCVF profiles under Scalings 1 and 2 (the legend applies to (A) only). (B) variation in the healthy rod:cone ratio, $\tilde{p}_{r}(\theta) / \tilde{p}_{c}(\theta)$, with eccentricity. (C) RdCVF production, consumption and decay rates under Scalings 1 and 2 (Eqn. (1), the legend applies to (C) only). To obtain $f_{\text {init }}(\theta)$ in (A) and (C), Eqs. (1) and (4) were solved at steady-state using the finite difference method, with 4001 mesh points, where $p_{r}(\theta)=p_{r_{\text {init }}}(\theta)$ and $p_{c}(\theta)=p_{c_{\text {init }}}(\theta)$. Under Scaling $1, \alpha=7.01 \times 10^{4}$ and $\beta=1.79 \times 10^{6}$, while under Scaling $2, \alpha=7.01 \times 10^{2}$ and $\beta=1.79 \times 10^{4}$. Remaining parameter values as in Table 2 . 
A

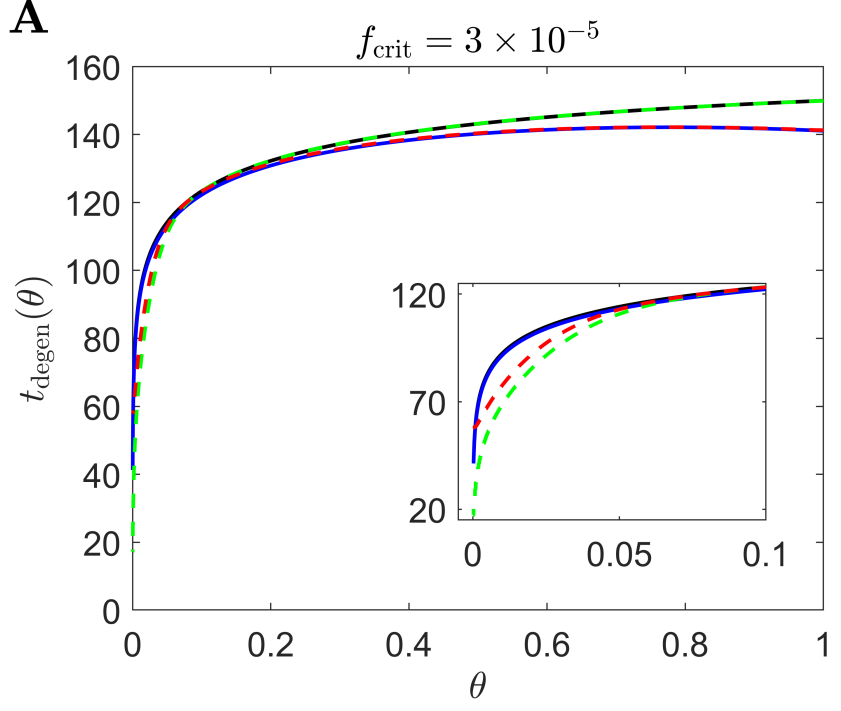

B

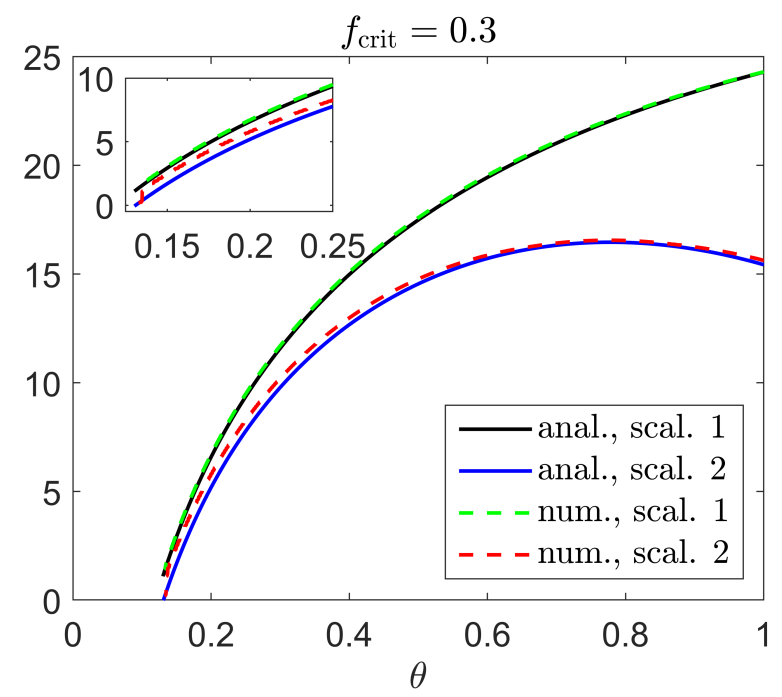

Figure 4. Cone degeneration profiles. Graphs show the time, $t_{\operatorname{degen}}(\theta)$, at which cones degenerate due to RdCVF deprivation, with constant rate of mutation-induced rod degeneration, $\phi_{r}=7.33 \times 10^{-2}$, and constant TF threshold concentrations: $f_{\text {crit }}=3 \times 10^{-5}(\mathbf{A})$ and $f_{\text {crit }}=0.3(\mathbf{B})$. The solid black and dashed green curves correspond to Scaling $1\left(\alpha=7.01 \times 10^{4}\right.$ and $\left.\beta=1.79 \times 10^{6}\right)$, while the solid blue and dashed red curves correspond to Scaling $2\left(\alpha=7.01 \times 10^{2}\right.$ and $\left.\beta=1.79 \times 10^{4}\right)$. The black and blue solid curves are analytical approximations, obtained by plotting Eqs. (6) and (9) respectively, while the green and red dashed curves are $p_{c}(\theta, t) / \tilde{p}_{c}(\theta)=0.99$ contours, obtained by solving Eqs. (1)-(5) using the method of lines with 401 mesh points. (A) simulation spans $\sim 17.7$ years in dimensional variables; $(\mathbf{B})$ simulation spans $\sim 2.8$ years in dimensional variables. Insets show magnified portions of each graph. Cone degeneration initiates at the fovea $(\theta=0)$ in $(\mathbf{A})$ and at $\theta=0.13$ in $(\mathbf{B})$, spreading peripherally (rightwards) in both cases. Degeneration occurs earlier in (B) than in (A) and for Scaling 2 than for Scaling 1 (except near the fovea in (A)). Remaining parameter values as in Table 2 .

Table 2. Parameters employed in the non-dimensional mathematical model (Eqs. (1)-(5)). Values are given to three significant figures (radians are dimensionless).

\begin{tabular}{lll}
\hline Parameter & Description & Value \\
\hline$\Theta$ & Eccentricity of the ora serrata & $1.33 \mathrm{rad}$ \\
$D_{f}$ & Trophic factor diffusivity & 0.237 \\
$\alpha$ & Rate of trophic factor production by rods & $7.01 \times 10^{2}$ or $7.01 \times 10^{4}$ \\
$\beta$ & Rate of trophic factor consumption by cones & $1.79 \times 10^{4}$ or $1.79 \times 10^{6}$ \\
$\eta$ & Rate of trophic factor decay & $1.79 \times 10^{2}$ \\
$\phi_{r}$ & Rate of mutation-induced rod degeneration & $7.33 \times 10^{-2}$ \\
$f_{\text {crit }}$ & Trophic factor threshold concentration & $3 \times 10^{-5}$ or 0.3 \\
$B_{1}$ & Cone profile parameter & 1.56 \\
$B_{2}$ & Cone profile parameter & 0.158 \\
$B_{3}$ & Rod profile parameter & 10.6 \\
$b_{1}$ & Cone profile parameter & 71.8 \\
$b_{2}$ & Cone profile parameter & 2.67 \\
$b_{3}$ & Rod profile parameter & 3.06 \\
\hline
\end{tabular}


bioRxiv preprint doi: https://doi.org/10.1101/2021.09.04.458968; this version posted February 14, 2022. The copyright holder for this preprint (which was not certified by peer review) is the author/funder, who has granted bioRxiv a license to display the preprint in perpetuity. It is made available under aCC-BY-NC-ND 4.0 International license.
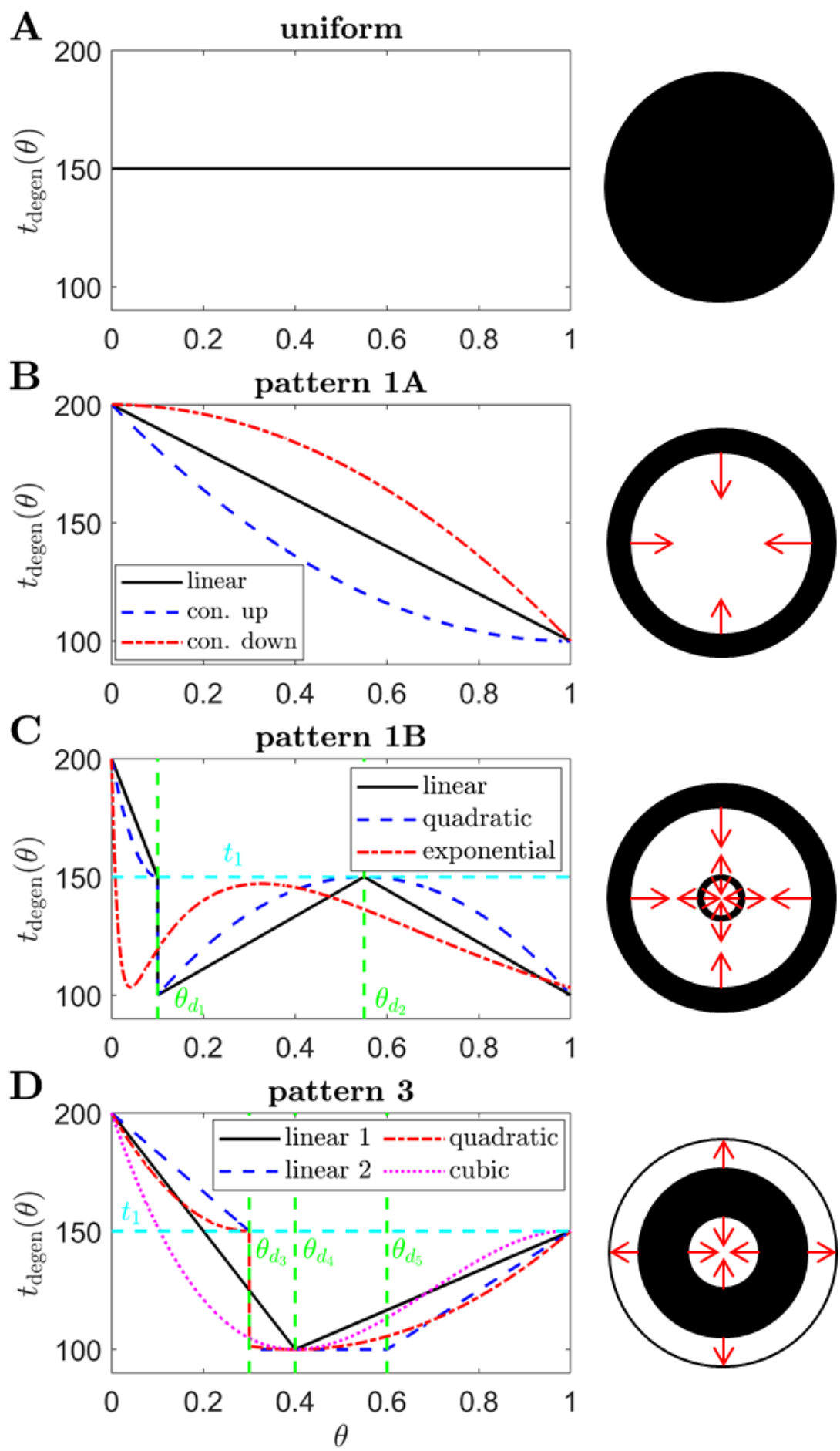

Figure 5. Target cone degeneration profiles. Panels (left) show cone degeneration profiles, $t_{\operatorname{degen}}(\theta)$, qualitatively replicating typical spatio-temporal patterns of visual field loss in RP: (A) Uniform, (B) Pattern 1A, (C) Pattern $1 \mathrm{~B}$ and (D) Pattern 3. Visual field loss patterns directly correspond to cone degeneration patterns in these radially symmetric cases. We seek to replicate these patterns by finding appropriate $\phi_{r_{\text {inv }}}(\theta)$ and $f_{\text {crit }_{\text {inv }}}(\theta)$ profiles in Figs. 679. Diagrams on the right show the corresponding 2D patterns of visual field loss - white regions: preserved vision, black regions: scotomas (blind spots), and red arrows: direction of scotoma propagation. Parameters: $t_{0}=100$ $(\sim 11.0$ years $), t_{1}=150(\sim 16.6$ years $), t_{2}=200\left(\sim 22.1\right.$ years, $\theta_{d_{1}}=0.1(\sim 7.6$ degrees $), \theta_{d_{2}}=0.55$ ( $\sim 41.9$ degrees $), \theta_{d_{3}}=0.3$ ( $\sim 22.9$ degrees $), \theta_{d_{4}}=0.4$ ( $\sim 30.5$ degrees $)$ and $\theta_{d_{5}}=0.6$ ( $\sim 45.7$ degrees $)$. Cone degeneration profile formulas and parameters are given in Table 3 . Remaining parameter values as in Table 2 . 
bioRxiv preprint doi: https://doi.org/10.1101/2021.09.04.458968; this version posted February 14, 2022. The copyright holder for this preprint (which was not certified by peer review) is the author/funder, who has granted bioRxiv a license to display the preprint in perpetuity. It is made available under aCC-BY-NC-ND 4.0 International license.

A



B



Figure 6. Inverse mutation-induced rod degeneration rate and TF threshold concentration - Uniform target cone degeneration profile. (A) inverse mutation-induced rod degeneration rate, $\phi_{r_{\text {inv }}}(\theta)\left(f_{\text {crit }}=3 \times 10^{-5}\right)$; (B) inverse TF threshold concentration, $f_{\text {crit }}$ inv $(\theta)\left(\phi_{r}=7.33 \times 10^{-2}\right)$. The solid black and dashed green curves correspond to Scaling $1\left(\alpha=7.01 \times 10^{4}\right.$ and $\left.\beta=1.79 \times 10^{6}\right)$, while the solid blue and dashed red curves correspond to Scaling $2\left(\alpha=7.01 \times 10^{2}\right.$ and $\left.\beta=1.79 \times 10^{4}\right)$. The black and blue solid curves are analytical approximations to the inverses, obtained by plotting Eqs. (7) and (10) respectively (A), and Eqs. (8) and (11) respectively (B). The green and red dashed curves are numerical inverses, obtained by using the Matlab routines fminsearch (A) and patternsearch (B) to calculate the $\phi_{r}$ and $f_{\text {crit }}$ profiles for which the contour described by $p_{c}(\theta, t) / \tilde{p}_{c}(\theta)=0.99$ matches the target cone degeneration profile, $t_{\text {degen }}(\theta)$. Eqs. (1)-(5) were solved at each iteration using the method of lines, with 101 mesh points. Insets show magnified portions of each graph. Numerical inverses are calculated and plotted only at those locations (eccentricities) where the analytical inverse fails to generate a $t_{\text {degen }}(\theta)$ profile matching the target profile. Inverses are monotone increasing for Scaling 1, and increase initially for Scaling 2 before reaching a maximum and decreasing toward the ora serrata $(\theta=1)$. Numerical solutions reveal lower values of the inverses near the fovea $(\theta=0)$ than the analytical approximations suggest. Cone degeneration profile formulas and parameters are given in Table 3. Remaining parameter values as in Table 2 
$\mathbf{A}$

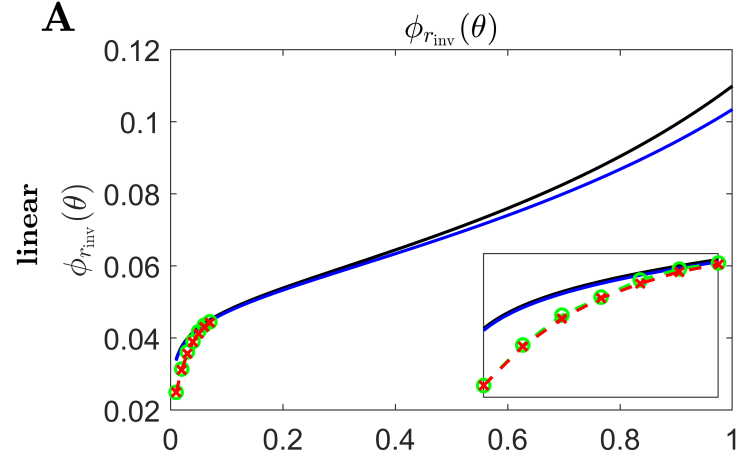

C

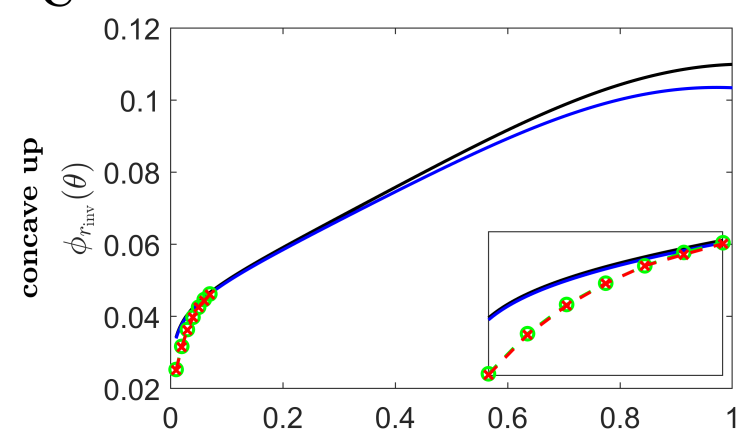

$\mathbf{E}$

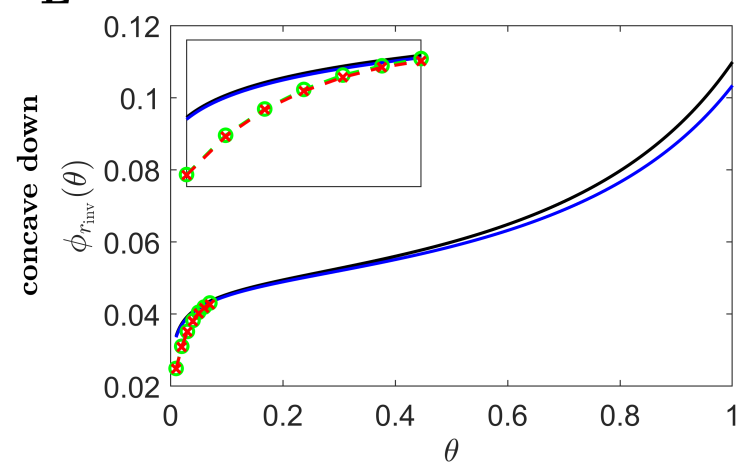

B

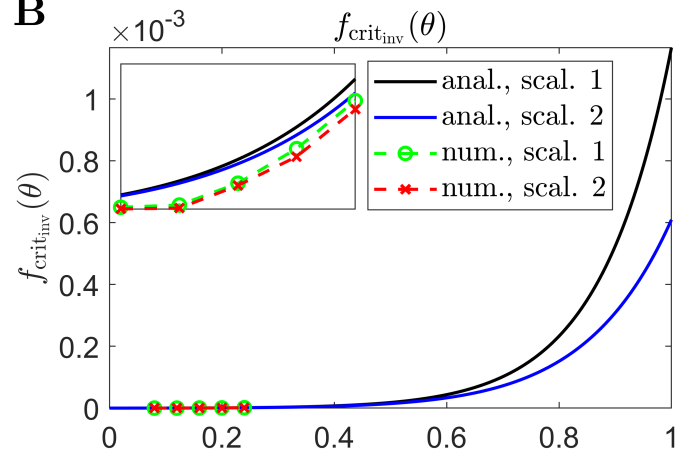

D

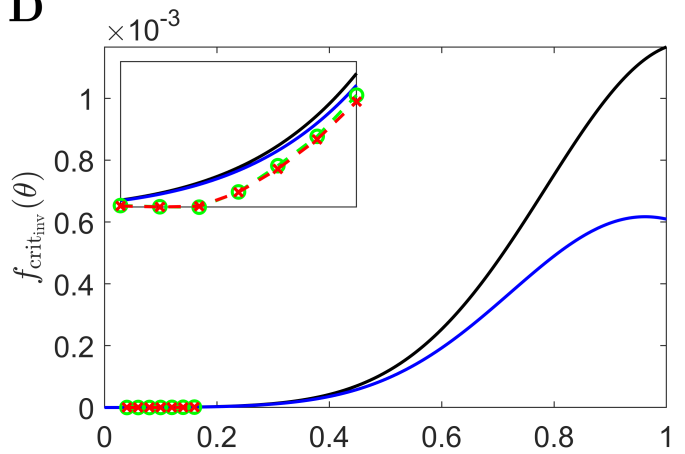

$\mathbf{F}$

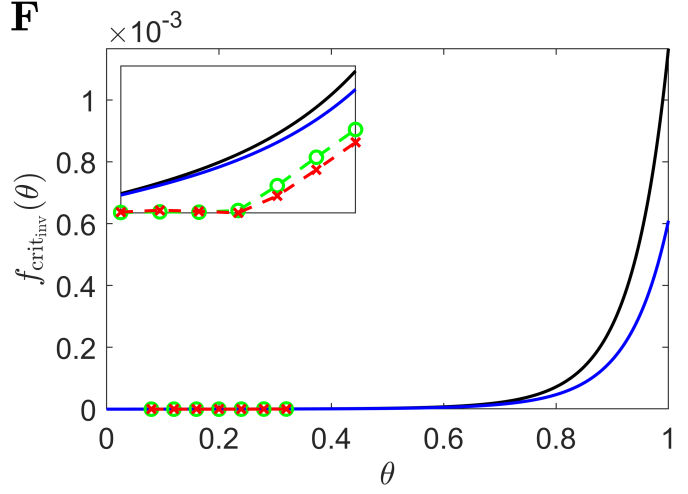

Figure 7. Inverse mutation-induced rod degeneration rate and $\mathrm{TF}$ threshold concentration - Pattern $1 \mathrm{~A}$ target cone degeneration profiles. (A), (C) and (E) inverse mutation-induced rod degeneration rate, $\phi_{r_{\text {inv }}}(\theta)\left(f_{\text {crit }}=3 \times 10^{-5}\right)$; (B), (D) and (F) inverse TF threshold concentration, $f_{\text {critinv }}(\theta)\left(\phi_{r}=7.33 \times 10^{-2}\right)$. (A) and (B) linear target cone degeneration profile, $t_{\text {degen }}(\theta)$; $(\mathbf{C})$ and $(\mathbf{D})$ concave up quadratic $t_{\text {degen }}(\theta)$ profile; $(\mathbf{E})$ and $(\mathbf{F})$ concave down quadratic $t_{\text {degen }}(\theta)$ profile. The solid black and dashed green curves correspond to Scaling $1\left(\alpha=7.01 \times 10^{4}\right.$ and $\left.\beta=1.79 \times 10^{6}\right)$, while the solid blue and dashed red curves correspond to Scaling $2\left(\alpha=7.01 \times 10^{2}\right.$ and $\left.\beta=1.79 \times 10^{4}\right)$. The black and blue solid curves are analytical approximations to the inverses, obtained by plotting Eqs. (7) and (10) respectively $(\mathbf{A}),(\mathbf{C})$ and $(\mathbf{E})$, and Eqs. (8) and (11) respectively (B), (D) and (F). The green and red dashed curves are numerical inverses, obtained by using the Matlab routines fminsearch (A), (C) and (E), and patternsearch (B), (D) and (F) to calculate the $\phi_{r}$ and $f_{\text {crit }}$ profiles for which the contour described by $p_{c}(\theta, t) / \tilde{p}_{c}(\theta)=0.99$ matches the target cone degeneration profile, $t_{\text {degen }}(\theta)$. Eqs. (1)-(5) were solved at each iteration using the method of lines, with 26, 51 or 101 mesh points. Insets show magnified portions of each graph. Numerical inverses are calculated and plotted only at those locations (eccentricities) where the analytical inverse fails to generate a $t_{\text {degen }}(\theta)$ profile matching the target profile. Inverses are monotone increasing functions for both scalings in (A), (B), (E) and (F), and for Scaling 1 in (C) and (D), while the inverses increase initially for Scaling 2 before reaching a maximum and decreasing toward the ora serrata $(\theta=1)$ in $(\mathbf{C})$ and $(\mathbf{D})$. Numerical solutions reveal lower values of the inverses near the fovea $(\theta=0)$ than the analytical approximations suggest. Cone degeneration profile formulas and parameters are given in Table 3 . Remaining parameter values as in Table 2. 
A

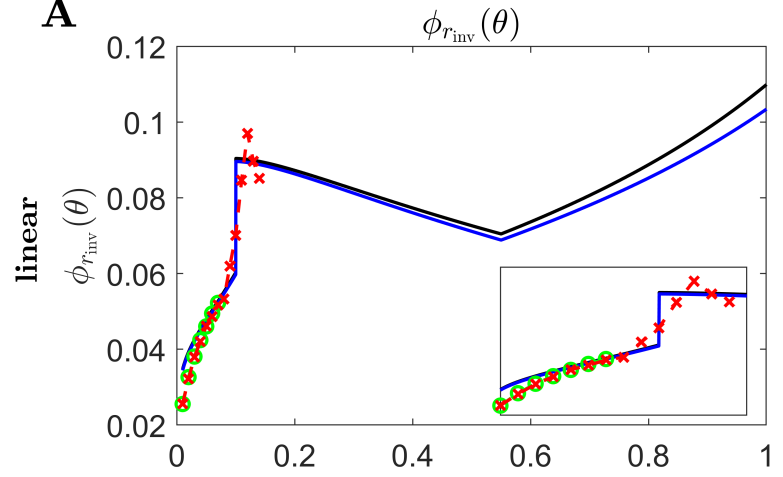

C

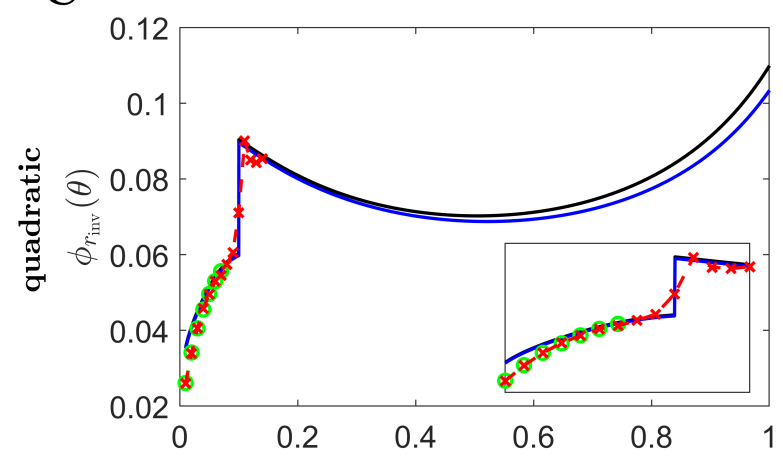

$\mathbf{E}$

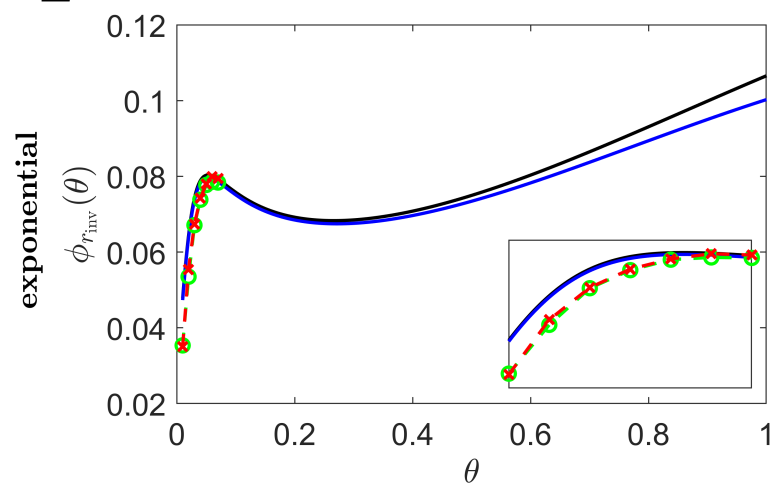

B

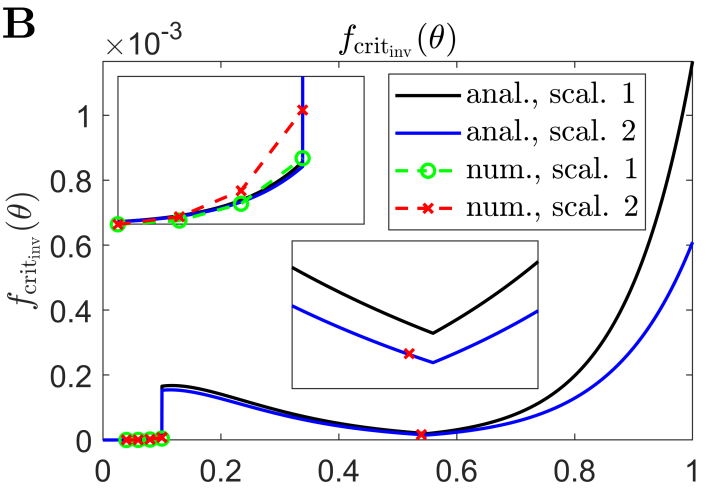

D

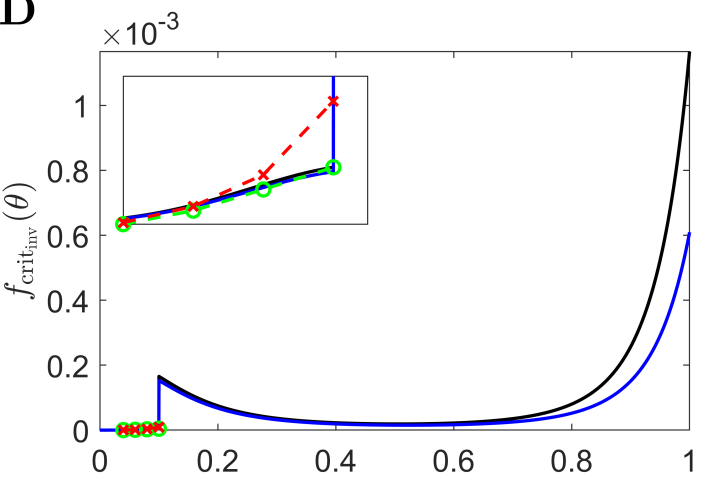

$\mathbf{F}$

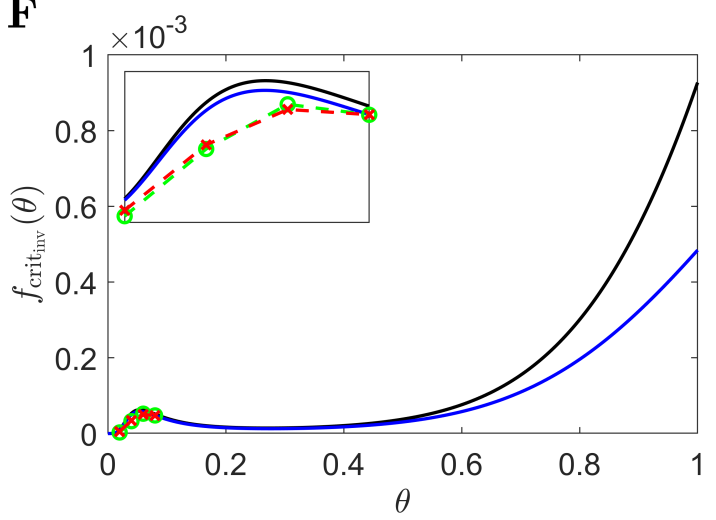

Figure 8. Inverse mutation-induced rod degeneration rate and TF threshold concentration - Pattern $1 \mathrm{~B}$ target cone degeneration profiles. (A), (C) and (E) inverse mutation-induced rod degeneration rate, $\phi_{r_{\text {inv }}}(\theta)\left(f_{\text {crit }}=3 \times 10^{-5}\right)$; (B), (D) and (F) inverse TF threshold concentration, $f_{\text {crit }}$ inv $_{1}(\theta)\left(\phi_{r}=7.33 \times 10^{-2}\right)$. (A) and (B) linear target cone degeneration profile, $t_{\text {degen }}(\theta)$; (C) and (D) quadratic $t_{\text {degen }}(\theta)$ profile; (E) and (F) exponential $t_{\text {degen }}(\theta)$ profile. The solid black and dashed green curves correspond to Scaling $1\left(\alpha=7.01 \times 10^{4}\right.$ and $\left.\beta=1.79 \times 10^{6}\right)$, while the solid blue and dashed red curves correspond to Scaling $2\left(\alpha=7.01 \times 10^{2}\right.$ and $\left.\beta=1.79 \times 10^{4}\right)$. The black and blue solid curves are analytical approximations to the inverses, obtained by plotting Eqs. (7) and (10) respectively (A), (C) and (E), and Eqs. (8) and (11) respectively (B), (D) and (F). The green and red dashed curves are numerical inverses, obtained by using the Matlab routines fminsearch (A), (C) and (E), and patternsearch (B), (D) and (F) to calculate the $\phi_{r}$ and $f_{\text {crit }}$ profiles for which the contour described by $p_{c}(\theta, t) / \tilde{p}_{c}(\theta)=0.99$ matches the target cone degeneration profile, $t_{\text {degen }}(\theta)$. Eqs. (1)-(5) were solved at each iteration using the method of lines, with 51 or 101 mesh points. Insets show magnified portions of each graph. Numerical inverses are calculated and plotted only at those locations (eccentricities) where the analytical inverse fails to generate a $t_{\text {degen }}(\theta)$ profile matching the target profile. Inverses resemble vertically flipped versions of the $t_{\operatorname{degen}}(\theta)$ profiles. Numerical solutions reveal lower values of the inverses near the fovea $(\theta=0)$ than the analytical approximations suggest and higher values in some regions away from the fovea in (A)-(D). Cone degeneration profile formulas and parameters are given in Table 3 . Remaining parameter values as in Table 2 . 

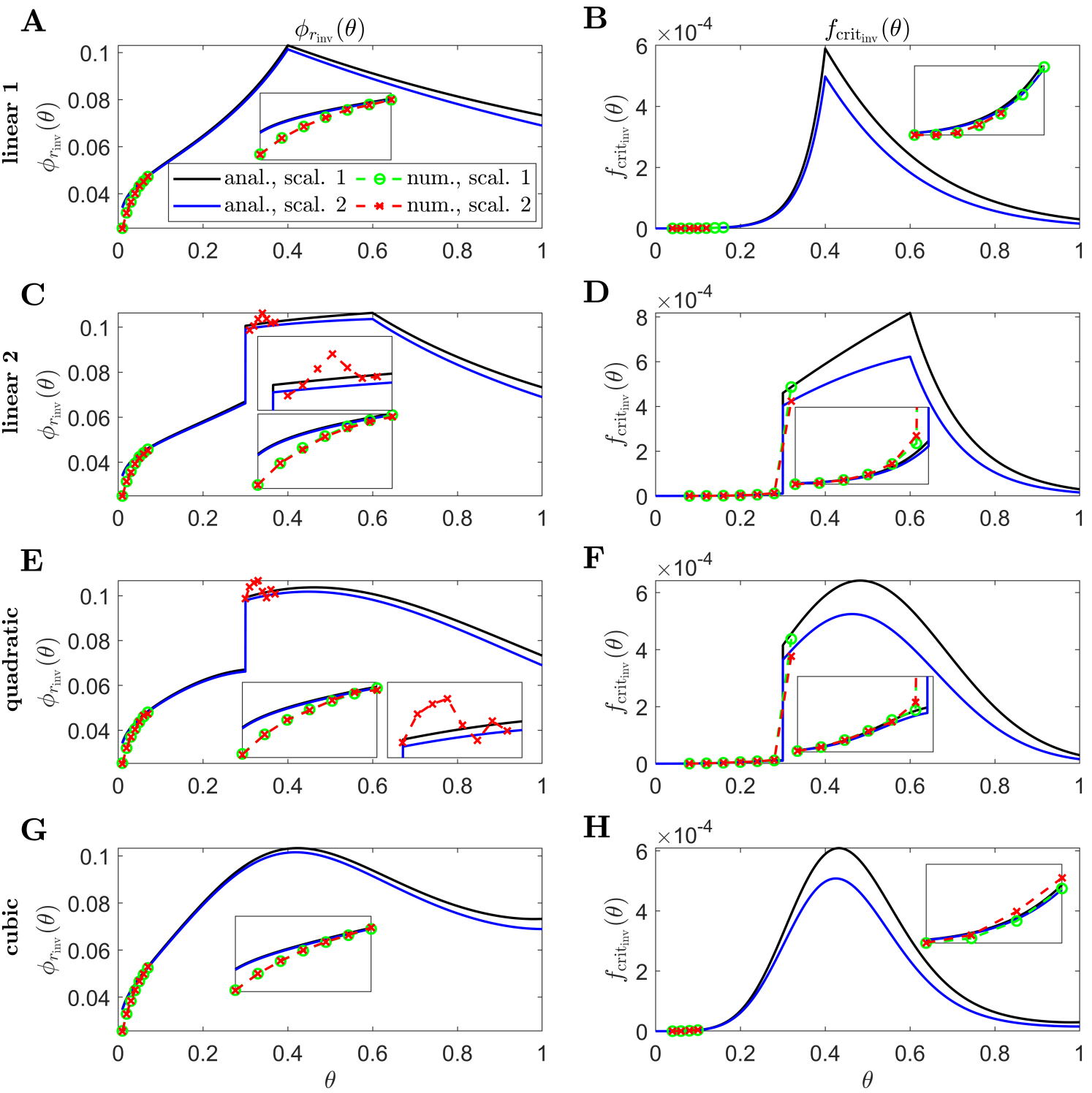

Figure 9. Inverse mutation-induced rod degeneration rate and TF threshold concentration - Pattern 3 target cone degeneration profiles. (A), (C), (E) and (G) inverse mutation-induced rod degeneration rate, $\phi_{r_{\text {inv }}}(\theta)\left(f_{\text {crit }}=\right.$ $\left.3 \times 10^{-5}\right)$; (B), (D), $(\mathbf{F})$ and $(\mathbf{H})$ inverse TF threshold concentration, $f_{\text {critinv }_{\text {inv }}}(\theta)\left(\phi_{r}=7.33 \times 10^{-2}\right)$. (A) and (B) linear 1 target cone degeneration profile, $t_{\text {degen }}(\theta)$; (C) and (D) linear $2 t_{\text {degen }}(\theta)$ profile; (E) and (F) quadratic $t_{\text {degen }}(\theta)$ profile; $(\mathbf{G})$ and $(\mathbf{H})$ cubic $t_{\text {degen }}(\theta)$ profile. The solid black and dashed green curves correspond to Scaling $1\left(\alpha=7.01 \times 10^{4}\right.$ and $\left.\beta=1.79 \times 10^{6}\right)$, while the solid blue and dashed red curves correspond to Scaling 2 $\left(\alpha=7.01 \times 10^{2}\right.$ and $\left.\beta=1.79 \times 10^{4}\right)$. The black and blue solid curves are analytical approximations to the inverses, obtained by plotting Eqs. (7) and (10) respectively (A), (C), (E) and (G), and Eqs. (8) and (11) respectively (B), (D), (F) and (H). The green and red dashed curves are numerical inverses, obtained by using the Matlab routines fminsearch (A), (C), (E) and (G), and patternsearch (B), (D), (F) and (H) to calculate the $\phi_{r}$ and $f_{\text {crit }}$ profiles for which the contour described by $p_{c}(\theta, t) / \tilde{p}_{c}(\theta)=0.99$ matches the target cone degeneration profile, $t_{\text {degen }}(\theta)$. Eqs. (1)-(5) were solved at each iteration using the method of lines, with 26, 51 or 101 mesh points. Insets show magnified portions of each graph. Numerical inverses are calculated and plotted only at those locations (eccentricities) where the analytical inverse fails to generate a $t_{\text {degen }}(\theta)$ profile matching the target profile. Inverses resemble vertically flipped versions of the $t_{\text {degen }}(\theta)$ profiles. Numerical solutions reveal lower values of the inverses near the fovea $(\theta=0)$ than the analytical approximations suggest and higher values in some regions away from the fovea in $(\mathbf{C})-(\mathbf{F})$ and $(\mathbf{H})$. Cone degeneration profile formulas and parameters are given in Table 3 . Remaining parameter values as in Table 2 
bioRxiv preprint doi: https://doi.org/10.1101/2021.09.04 458968; this version posted February 14,2022 . The copyright holder for this preprint (which was not certified by peer review) is the author/funder, who has granted bioRxiv a license to display the preprint in perpetuity. It is made available under aCC-BY-NC-ND 4.0 International license.
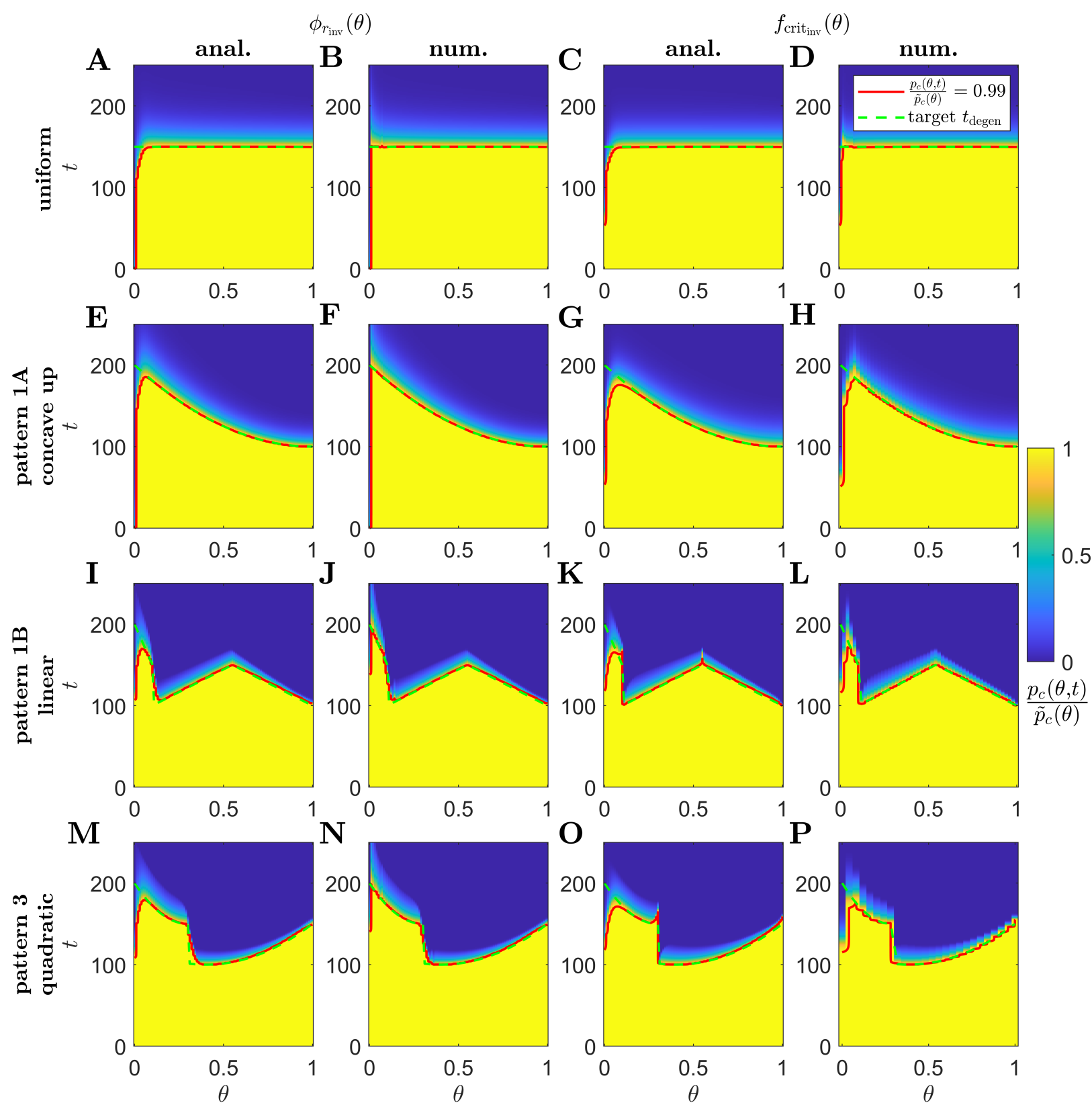

Figure 10. Simulations of proportional cone loss for a range of inverse mutation-induced rod degeneration rates and TF threshold concentrations. Plots show the proportion of cones remaining compared to local healthy values, $p_{c}(\theta, t) / \tilde{p}_{c}(\theta)$, across space and over time. (A), (E), (I) and (M) analytical inverse mutation-induced rod degeneration rate, $\phi_{r_{\text {inv }}}(\theta)\left(f_{\text {crit }}=3 \times 10^{-5}\right) ;(\mathbf{B}),(\mathbf{F}),(\mathbf{J})$ and $(\mathbf{N})$ numerical $\phi_{r_{\text {inv }}}(\theta)\left(f_{\text {crit }}=3 \times 10^{-5}\right) ;(\mathbf{C}),(\mathbf{G})$, $(\mathbf{K})$ and $(\mathbf{O})$ analytical inverse TF threshold concentration, $f_{\text {crititiv }}(\theta)\left(\phi_{r}=7.33 \times 10^{-2}\right) ;(\mathbf{D}),(\mathbf{H}),(\mathbf{L})$ and $(\mathbf{P})$ numerical $f_{\text {crit }_{\text {inv }}}(\theta)\left(\phi_{r}=7.33 \times 10^{-2}\right)$. (A)-(D) Uniform target cone degeneration profile, $t_{\text {degen }}(\theta)$, with Scaling $1\left(\alpha=7.01 \times 10^{4}\right.$ and $\left.\beta=1.79 \times 10^{6}\right) ;(\mathbf{E})-(\mathbf{H})$ Pattern 1A quadratic concave up $t_{\text {degen }}(\theta)$ profile with Scaling 1 ; (I) $-(\mathbf{L})$ Pattern 1B linear $t_{\text {degen }}(\theta)$ profile with Scaling $2\left(\alpha=7.01 \times 10^{2}\right.$ and $\left.\beta=1.79 \times 10^{4}\right)$; (M)-(P) Pattern 3 quadratic $t_{\text {degen }}(\theta)$ profile with Scaling 2. Eqs. (1)-(5) were solved using the method of lines, with 26, 51 or 101 mesh points. Analytical and numerical $\phi_{r_{\text {inv }}}(\theta)$ and $f_{\text {crit }_{\text {inv }}}(\theta)$ are as plotted in Figs. 6- 9. Solid red curves denote the contours along which $p_{c}(\theta, t) / \tilde{p}_{c}(\theta)=0.99$, while dashed green curves show the target $t_{\text {degen }}(\theta)$ profiles. Cone degeneration profiles generally show good agreement with the target $t_{\text {degen }}(\theta)$ profiles. There is some divergence from $t_{\text {degen }}(\theta)$ for the analytical inverses near the fovea $(\theta=0)$ and at discontinuous or nonsmooth portions of $t_{\text {degen }}(\theta)$; this is mostly corrected by the numerical inverses. Cone degeneration profile formulas and parameters are given in Table 3 . Remaining parameter values as in Table 2. 
bioRxiv preprint doi: https://doi org/10.1101/2021.09.04.458968; this version posted February $14,2022$. The copyright holder for this preprint (which was not certified by peer review) is the author/funder, who has granted bioRxiv a license to display the preprint in perpetuity. It is made available under aCC-BY-NC-ND 4.0 International license.

Table 3. Target cone degeneration profiles, $t_{\mathrm{degen}}(\theta)$.

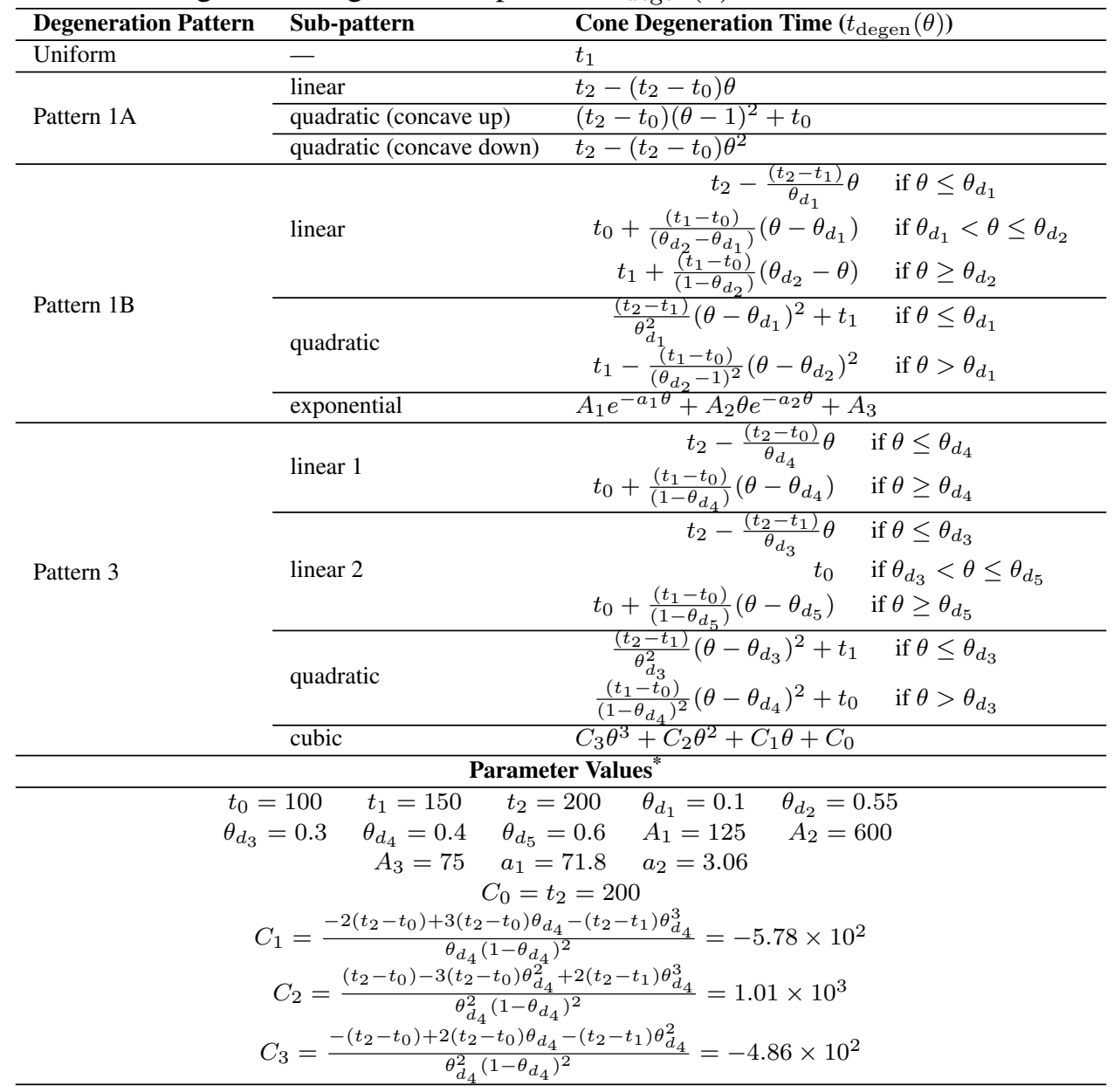

* We choose $\theta_{d_{1}}$ and $\theta_{d_{2}}$ such that $\theta_{d_{2}}=\left(\theta_{d_{1}}+1\right) / 2$, so that $\theta_{d_{2}}$ lies halfway between $\theta=\theta_{d_{1}}$ and $\theta=1$. 